\title{
DO DISTINCT COSMOLOGICAL MODELS PREDICT DEGENERATE HALO POPULATIONS?
}

\author{
Zheng Zheng, ${ }^{1}$ Jeremy L. Tinker, ${ }^{1}$ David H. Weinberg, ${ }^{1}$ And Andreas A. Berlind ${ }^{2}$ \\ Received 2002 February 19; accepted 2002 April 25
}

\begin{abstract}
Using cosmological $N$-body simulations, we investigate the influence of the matter density parameter $\Omega_{m}$ and the linear theory power spectrum $P(k)$ on statistical properties of the dark matter halo population-the mass function $n(M)$, two-point correlation function $\xi(r)$, and pairwise velocity statistics $v_{12}(r)$ and $\sigma_{12}(r)$. For fixed linear theory $P(k)$, the effect of changing $\Omega_{m}$ is simple: the halo mass scale $M_{*}$ shifts in proportion to $\Omega_{m}$, pairwise velocities (at fixed $M / M_{*}$ ) are proportional to $\Omega_{m}^{0.6}$, and halo clustering at fixed $M / M_{*}$ is unchanged. If one simultaneously changes the power spectrum amplitude $\sigma_{8}$ to maintain the "cluster normalization " condition $\sigma_{8} \Omega_{m}^{0.5}=$ const, then $n(M)$ stays approximately constant near $M \sim 5 \times 10^{14} h^{-1} M_{\odot}$, and halo clustering and pairwise velocities are similar at fixed $M$. However, the shape of $n(M)$ changes, with a decrease of $\Omega_{m}$ from 0.3 to 0.2 , producing a $\sim 30 \%$ drop in the number of low-mass halos. One can preserve the shape of $n(M)$ over a large dynamic range by changing the spectral tilt $n_{s}$ or shape parameter $\Gamma$, but the required changes are substantial-e.g., masking a decrease of $\Omega_{m}$ from 0.3 to 0.2 requires $\Delta n_{s} \approx 0.3$ or $\Delta \Gamma \approx 0.15$. These changes to $P(k)$ significantly alter the halo clustering and halo velocities. The sensitivity of the dark halo population to cosmological model parameters has encouraging implications for efforts to constrain cosmology and galaxy bias with observed galaxy clustering, since the predicted changes in the halo population cannot easily be masked by altering the way that galaxies occupy halos. A shift in $\Omega_{m}$ alone would be detected by any dynamically sensitive clustering statistic; a cluster normalized change to $\sigma_{8}$ and $\Omega_{m}$ would require a change in galaxy occupation as a function of $M / M_{*}$, which would alter galaxy clustering; and a simultaneous change to $P(k)$ that preserves the halo mass function would change the clustering of the halos themselves.
\end{abstract}

Subject headings: cosmology: theory — dark matter — galaxies: formation — galaxies: halos — large-scale structure of universe

\section{INTRODUCTION}

In cosmological models dominated by cold dark matter (CDM) and an unclustered energy component (such as a cosmological constant), gravitational instability of primordial density fluctuations produces a population of dark matter halos, each in approximate virial equilibrium. Depending on their mass, these halos may host individual galaxies, galaxy groups, or rich galaxy clusters. Provided that one focuses on systems of overdensity $\rho / \bar{\rho} \sim 200$, and regards higher density structures as subsystems within their parent halos, one finds that the halo population itself is insensitive to the gas pressure forces that influence the subdominant baryon component. In this paper, we investigate whether changes to cosmological parameters - specifically the matter density parameter $\Omega_{m}$ and parameters that describe the shape and amplitude of the primordial power spectrum - always produce measurable changes in the dark halo population, or whether two models with different combinations of these parameters can give rise to halo populations that are effectively indistinguishable.

Our interest in this question is spurred by recent developments in the theory of biased galaxy formation. The uncertain relation between galaxies and dark matter is the primary limitation in testing cosmological models against observations of galaxy clustering. The "halo occupation

\footnotetext{
${ }^{1}$ Department of Astronomy, Ohio State University, Columbus, OH 43210; zhengz@astronomy.ohio-state.edu, tinker@astronomy.ohio-state.edu,dhw@astronomy.ohio-state.edu.

${ }^{2}$ Department of Astronomy and Astrophysics, The University of Chicago, Chicago, IL 60637; aberlind@oddjob.uchicago.edu.
}

distribution" (HOD) characterizes this relation statistically in terms of the probability distribution $P(N \mid M)$ that a halo of virial mass $M$ contains $N$ galaxies of a specified type, together with prescriptions that specify the relative spatial and velocity distributions of galaxies and dark matter within these halos. Numerous recent papers have shown that the HOD framework is a powerful tool for analytic and numerical calculations of clustering statistics, for modeling observed clustering, and for characterizing the results of semianalytic or numerical studies of galaxy formation (e.g., Kauffmann, Nusser, \& Steinmetz 1997; Jing, Mo, \& Börner 1998; Kauffman et al. 1999; Benson et al. 2000; Ma \& Fry 2000; Peacock \& Smith 2000; Seljak 2000; Berlind \& Weinberg 2002; Bullock, Wechsler, \& Somerville 2002; Marinoni \& Hudson 2002; Scoccimarro et al. 2001; Yoshikawa et al. 2001; White, Hernquist, \& Springel 2001). In particular, Peacock \& Smith (2000), Marinoni \& Hudson (2002), and Berlind \& Weinberg (2002) have argued that the HOD can be determined empirically from observed galaxy clustering, given an assumed cosmological model that determines the mass function and spatial and velocity clustering of the dark halo population. Empirical determinations of the HOD can provide insight into the physics of galaxy formation, and they may sharpen the ability of large-scale structure studies to test cosmological models, since a model with an incorrect dark halo population may be unable to match the data for any choice of HOD (see Berlind \& Weinberg 2002 for further discussion).

Suppose that we find a combination of cosmology and HOD that reproduces all aspects of observed galaxy clustering. Can we infer that the cosmology and the derived HOD 
are both correct? If there is another cosmological model that predicts an indistinguishable halo population, then the answer is clearly no, since the combination of this alternative cosmology with the same HOD would predict identical galaxy clustering. These cosmological models would be degenerate in the sense that they could not be distinguished by galaxy clustering data without relying on a predictive theory of galaxy formation (which might yield different HODs for the two cosmologies). Cosmological models that predict distinguishable halo populations could still be degenerate in this sense, if changes to the HOD can mask the differences in the halo populations; we will speculate on this point in $\S 4$, but we reserve a quantitative examination of it to future work.

We focus our analyses on CDM cosmological models with Gaussian initial density fluctuation fields. Motivated by cosmic microwave background measurements (Netterfield et al. 2002; Pryke et al. 2002), we restrict our attention to spatially flat models with a cosmological constant or $\Omega_{m}=1$; however, we demonstrate in passing that galaxy clustering data at $z=0$ probably cannot distinguish a flat model from an open model with the same $\Omega_{m}$. The parameters that define our cosmological models are the matter density parameter $\Omega_{m}$; the normalization $\sigma_{8}$ of the power spectrum $P(k)$, which is the rms fluctuation of the linear density field filtered with a top-hat filter of radius $8 h^{-1} \mathrm{Mpc}$; and the shape of $P(k)$, which we characterize by the spectral index $n_{s}$ of the inflationary power spectrum and the shape parameter $\Gamma$ of the matter transfer function (see, e.g., Efstathiou, Bond, \& White 1992). Although existing data impose constraints on these parameters, individually or in combination, here we allow each to vary independently over a fairly broad range so that we can isolate the physical effects of the matter density, the amplitude of mass fluctuations, and the shape of the power spectrum on the resulting halo population. We concentrate entirely on the halo populations at $z=0$, since this is where galaxy clustering data will be good enough to allow empirical HOD determinations in the near future.

Using $N$-body simulations described in $\S 2$, we measure (in $\S 3$ ) mass functions, two-point correlation functions, mean pairwise radial velocities, and pairwise velocity dispersions of dark matter halos. First, we consider models that have the same initial power spectrum $P(k)$ but different matter density parameter $\Omega_{m}$. We then move to cluster normalized models in which $\sigma_{8}$ is changed to compensate the change in $\Omega_{m}$ so that the amplitude of the halo mass function is kept approximately fixed at a cluster scale. Finally, we investigate models in which both the amplitude and the shape of $P(k)$ are changed in order to match the amplitude and slope of the halo mass function at the cluster scale. We review our results and briefly discuss their implications for cosmological tests in $\S 4$. Our results overlap previous numerical studies of the halo mass function and halo clustering (recent examples include Jing 1998; Governato et al. 1999; Colberg et al. 2000; Jenkins et al. 2001), but they differ in the examination of controlled parameter sequences rather than specific cosmological models. Also, while some of these studies have focused on the large-scale correlations of cluster mass halos, we devote considerable attention to the lower masses and smaller spatial scales that are important for nonlinear galaxy clustering. Throughout the paper, we adopt a Hubble constant $H_{0}=100 h \mathrm{~km} \mathrm{~s}^{-1} \mathrm{Mpc}^{-1}=$ $70 \mathrm{~km} \mathrm{~s}^{-1} \mathrm{Mpc}^{-1}$ (Freedman et al. 2001).

\section{NUMERICAL METHODS AND TESTS}

In this work, statistics are mainly based on simulations run with a particle-mesh (PM) N-body code (Park 1990). We run PM simulations with different combinations of density and power spectrum parameters. Each simulation follows the evolution of $200^{3}$ particles in a periodic cube 200 $h^{-1} \mathrm{Mpc}$ on a side, with a $400^{3}$ mesh to compute the gravitational force starting at $z=19$ and advancing to $z=0$ in 40 equal steps of expansion factor $a$. The mass of each particle is $2.78 \times 10^{11} \Omega_{m} h^{-1} M_{\odot}$. We adopt the parameterization of Efstathiou et al. (1992) for the fluctuation power spectrum $P(k)$. We divide our simulations into three categories corresponding to parameter changes in the matter density and the normalization and the shape of the initial fluctuation power spectrum. For each category, four cosmological models are investigated with $\left(\Omega_{m}, \Omega_{\Lambda}\right)=(0.2,0.8),(0.3$, $0.7),(0.4,0.6)$ and $(1.0,0.0)$. We generally regard the $(0.3$, $0.7)$ model as the central one for comparison. In the central model, the spectral index is $n_{s}=1.0$, the spectral shape parameter is $\Gamma=0.20$, and the rms mass fluctuation within spheres of radius $8 h^{-1} \mathrm{Mpc}$ is $\sigma_{8}=0.9$, which is consistent with the observed abundance of clusters (Eke, Cole, \& Frenk 1996). For each model, we generate four independent realizations, and we use the dispersion among these realizations to estimate the statistical error bars on our clustering measures associated with the finite simulation volume. The total volume is comparable to that expected for the Sloan Digital Sky Survey (York et al. 2000) out to its median redshift $z_{\text {med }} \approx 0.1$. We use a friends-of-friends (FOF) algorithm (Davis et al. 1985) to identify the dark matter halos, and unless explicitly stated otherwise, the linking length is 0.2 times the mean interparticle separation. With this linking length, the FOF algorithm picks out structures of typical mass overdensity $\rho / \bar{\rho} \sim 200$ (Davis et al. 1985).

The advantage of a PM code is that it is relatively cheap to run multiple simulations with a large dynamic range in mass. The limitation of a PM code is its moderate force resolution, but this limitation is not too serious for our purposes, since we only identify halos and do not attempt to measure their internal structure. We perform two tests to make sure that the PM resolution is adequate for our purpose. First, we make a comparison between PM simulations and simulations run with the GADGET tree code (Springel, Yoshida, \& White 2001). Second, we perform a self-similar scaling test for the PM code.

GADGET is a publicly available $N$-body code that calculates particle accelerations by the hierarchical tree method of Barnes \& Hut (1986). Its advantages over the PM algorithm are its spatial and temporal adaptability. The gravitational softening length $\epsilon$ can be set significantly smaller than the initial particle grid spacing to increase force resolution; however, if $\epsilon$ is too small, two-body relaxation effects will begin to influence the halo population. Particles have individual timesteps, which can vary continuously to ensure accurate time integration given the adopted force resolution. GADGET has fully periodic boundary conditions for comoving integrations via the Ewald summation method (Hernquist, Bouchet, \& Suto 1991).

We have compared the results of two simulations with $100^{3}$ particles in a $100 h^{-1} \mathrm{Mpc}$ box, one evolved with the PM code using a $200^{3}$ mesh (thus having equal resolution to the larger simulations on which our results will be based), and one evolved with GADGET using $\epsilon=0.3 h^{-1} \mathrm{Mpc}$. 
The initial conditions of the two simulations were identical to each other and used the parameters of the central model described above. Slices through these simulations are plotted for comparison in Figure 1. The structures formed in the two simulations are very similar at the level of detail discernible in this plot. The halo mass functions of the two simulations, also shown in Figure 1, trace each other nearly exactly. The PM simulation produces slightly $(\sim 10 \%)$ fewer halos below $10^{13} h^{-1} M_{\odot}$, which is to be expected given its lower force resolution; the diameter of an overdensity 200 sphere of $10^{13} h^{-1} M_{\odot}$ is $1.04 h^{-1} \mathrm{Mpc}$, or about two PM grid cells. When the mesh size on this PM simulation was increased to $300^{3}$, the resulting mass function was consistent with the GADGET simulation even on these small-mass scales.

In addition to the halo mass function, the quantities important to our analysis are the correlation functions for both the mass distributions and the halo populations, which are shown in Figure 2. As with the halo mass functions, the matter correlation functions for the PM and GADGET simulations match very well. They begin to deviate at separations smaller than $\sim 0.5 h^{-1} \mathrm{Mpc}$, or under one mesh cell.
The halo correlation functions produced by the two methods are also consistent. Larger simulations and multiple realizations of each model are efficiently produced with the PM code, which takes up to an order of magnitude less computing time at the force resolution adopted here.

We next perform a self-similar scaling test for the PM code. Assuming an Einstein-de Sitter universe $\left(\Omega_{m}=1\right)$, we use a pure power-law power spectrum $P(k) \propto k^{-n_{s}}$ with $n_{s}=-1$ (and no transfer function modulation) for the initial density fluctuations of the scale-free model. A $400^{3}$ force mesh is used to follow the gravitational evolution of $200^{3}$ particles. The normalization of the power spectrum is set so that the nonlinear mass $M_{*}$ at the final output corresponds to 400 particles. We define $M_{*}$ by the condition $\sigma\left(M_{*}\right)=\delta_{c}=1.69$, where $\sigma\left(M_{*}\right)$ is the rms linear mass fluctuation on mass scale $M_{*}$. The rms fluctuation is $\sigma=1.0$ at about $1 / 26$ of the box size. If we identify this scale as $8 h^{-1} \mathrm{Mpc}$, the implied size of the periodic simulation cube is then $207.62 h^{-1} \mathrm{Mpc}$ at $z=0$. Earlier outputs can be identified with higher redshifts or with larger volumes at $z=0$. In the language of the redshift description, the simulation is evolved for 40 time steps from $z=19$, with four
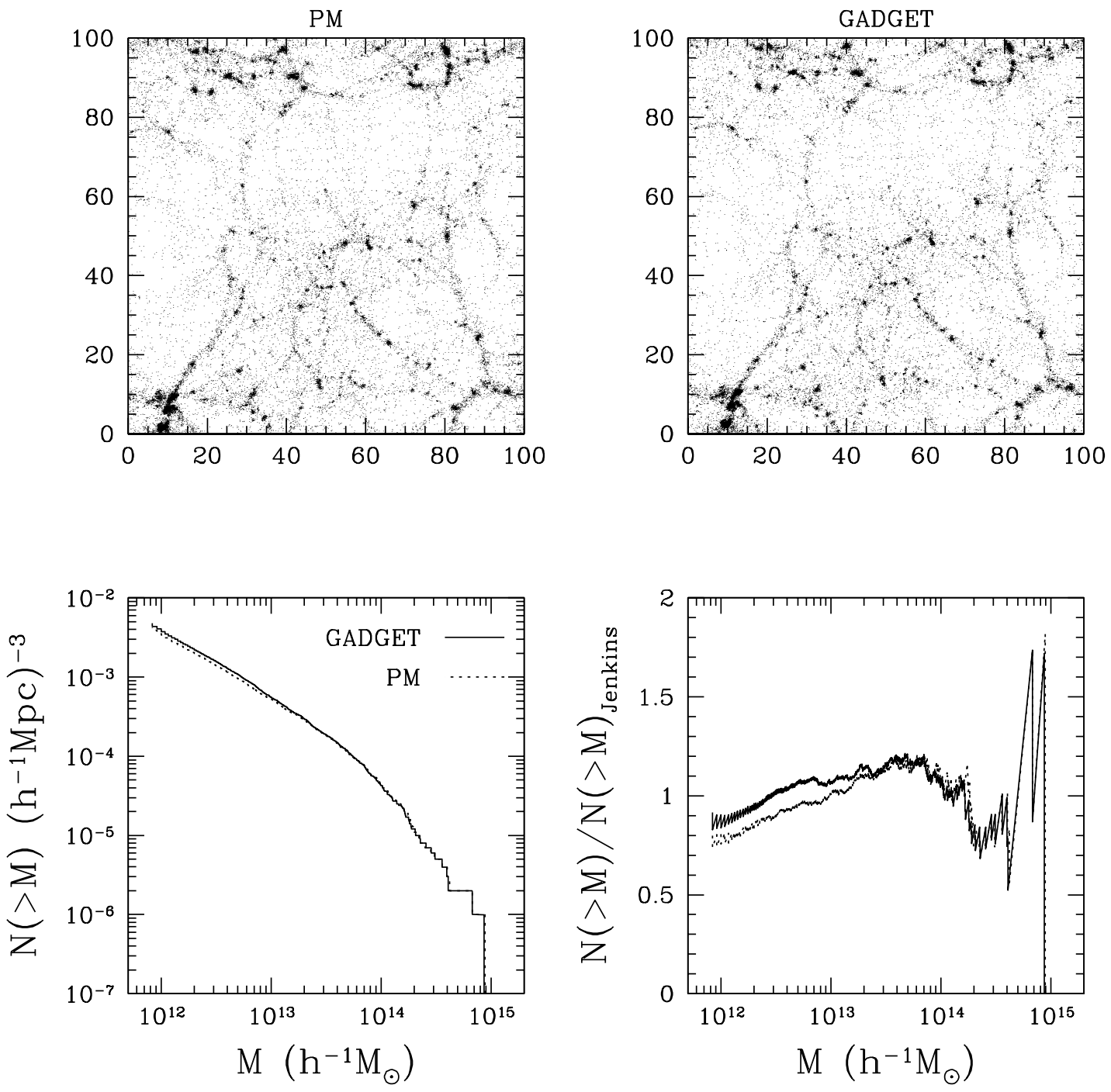

FIG. 1.-Comparison of PM and GADGET simulations evolved from the same initial conditions. Top panels show slices through the particle distributions, $100 \mathrm{~h}^{-1} \mathrm{Mpc}$ on a side and $10 \mathrm{~h}^{-1} \mathrm{Mpc}$ thick. Bottom panels show cumulative halo mass functions; in the right panel these are divided by the Jenkins et al. (2001) analytic mass function to allow more detailed comparison. The minimum group mass plotted corresponds to 10 simulation particles. 

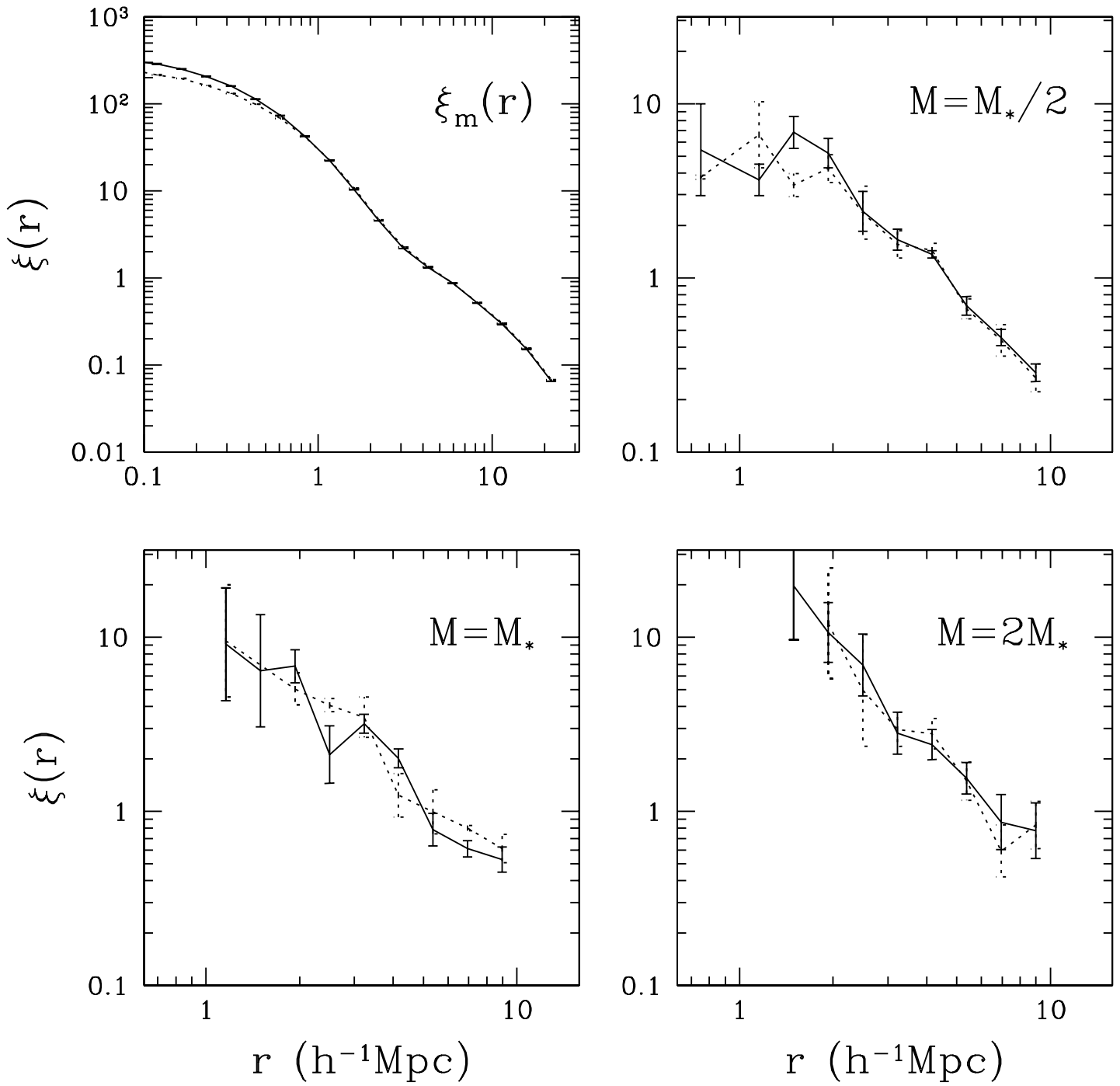

Fig. 2.-Matter and halo correlation functions of the PM (dotted lines) and GADGET (solid lines) simulations shown in Fig. 1. The upper left panel shows the matter correlation functions, and remaining panels show halo correlation functions for three different mass ranges, centered on $M_{*} / 2, M_{*}$, and $2 M_{*}$, where $M_{*}=1.03 \times 10^{13} h^{-1} M_{\odot}$ is the characteristic nonlinear mass (corresponding to 123 particles in these simulations). Results are averaged over four realizations, and error bars show the run-to-run dispersion divided by $\sqrt{N-1}=\sqrt{3}$ to yield the uncertainty in the mean.

outputs (at redshifts $z=1.00,0.60,0.25$, and 0.00 ), when the corresponding $M_{*}$ values are 50,100, 200, and 400 particles, respectively. The following results are averaged over four independent realizations of the simulation, and the error bars represent the dispersion among the four realizations divided by $\sqrt{3}$ to yield the error on the mean.

Efstathiou et al. (1988) performed the first study on scalefree cosmological models using $N$-body simulations and showed that many quantities in scale-free models have welldefined scaling behaviors. In an Einstein-de Sitter scale-free model, the cumulative halo mass functions at redshifts $z_{1}$ and $z_{2}$ have the scaling behavior

$$
\begin{aligned}
N\left(M>M_{2} ; z_{2}\right) & =\left(\frac{1+z_{2}}{1+z_{1}}\right)^{6 /\left(3+n_{s}\right)} N\left(M>M_{1} ; z_{1}\right), \\
M_{1} & =\left(\frac{1+z_{2}}{1+z_{1}}\right)^{6 /\left(3+n_{s}\right)} M_{2} .
\end{aligned}
$$

The left panel of Figure 3 shows the cumulative halo mass function at different redshifts in our scale-free simulations (solid curves). The dotted curves in Figure 3 are derived by scaling the mass function at $z=0$ according to the above scaling rule. The scaling properties shown in the figure are generally very good. The high-mass end of the mass function drops systematically below the self-similar scaling as $M_{*}$ increases, a likely result of the finite simulation volume, which suppresses the amplitude of fluctuations on the largest scales. Comparison of the first and last outputs suggests that this effect becomes noticeable above $M \sim 2 \times 10^{15}$ $h^{-1} M_{\odot}$ at $z=0$ (for our adopted $\sigma_{8}=1.0$ scaling), or a mass scale $\sim 0.1 \%$ of the total simulation mass $(10 \%$ in length scale).

The $N$-point correlation functions $\xi(r)$ are expected to have a similarity transformation with the variable $s=r(1+z)^{2 /\left(3+n_{s}\right)}$ for an Einstein-de Sitter universe (Efstathiou et al. 1988). The scaling rule for the correlation functions at redshifts $z_{1}$ and $z_{2}$ is then

$$
\xi\left(r_{2} ; z_{2}\right)=\xi\left(r_{1} ; z_{1}\right), \quad r_{1}=\left(\frac{1+z_{2}}{1+z_{1}}\right)^{2 /\left(3+n_{s}\right)} r_{2} .
$$



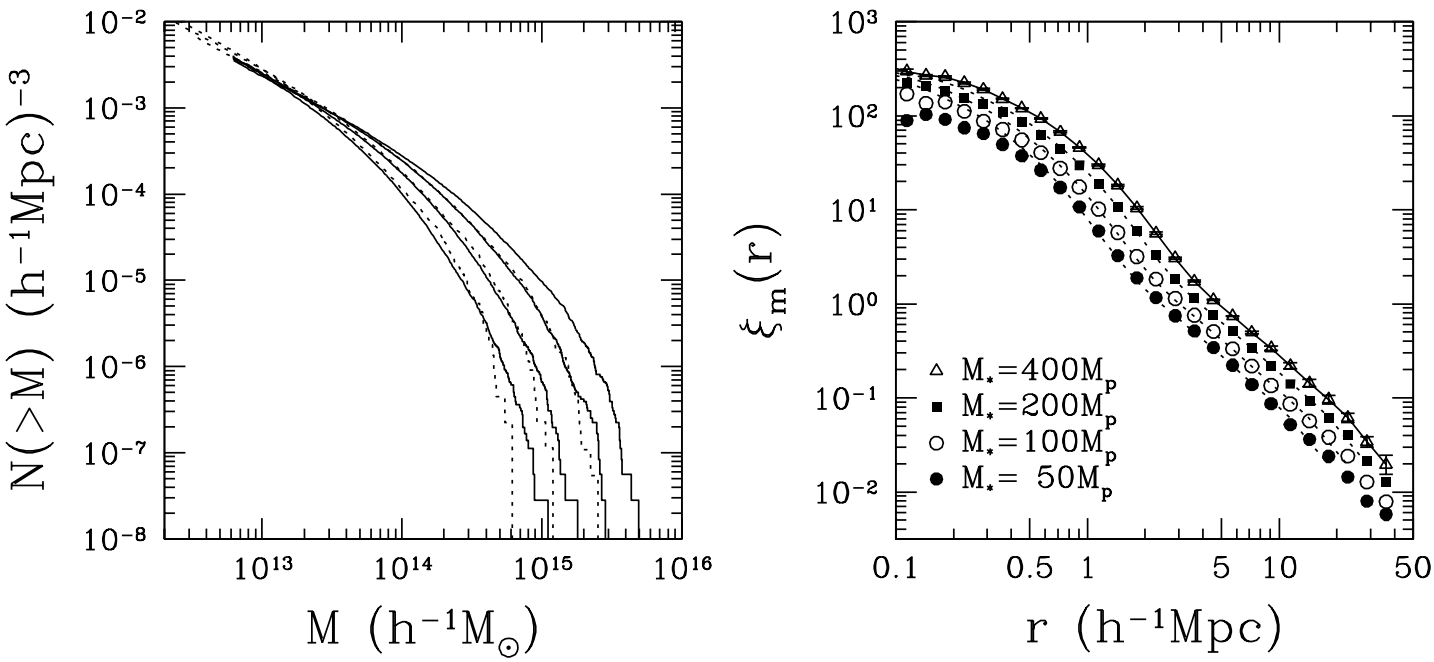

FIG. 3.-Test of self-similar scaling in PM simulations with scale-free initial conditions. Solid lines in the left panel show halo mass functions at four evolutionary stages, when $M_{*}=50,100,200$, and 400 particles ( from left to right). Dotted lines show the rightmost solid line scaled according to eq. (1). Points in the right panel show two-point correlation functions of the mass at these four epochs. Dotted lines are derived by scaling the mass correlation function of the final output, when $M_{*}=400 M_{p}$, according to eq. (2). Results are averaged over four simulations, and error bars on $\xi(r)$ show the dispersion among four independent runs, divided by $\sqrt{3}$. For clarity, error bars are plotted only for the stage of $M_{*}=400 M_{p}$, and error bars for other stages have similar magnitude. Physical scales are assigned by treating the final output as $z=0$ and adopting $\sigma_{8}=1.0$, which implies a comoving size $L_{\mathrm{box}}=207.62 h^{-1} M_{\odot}$ of the simulation cube and a total mass $M=2.49 \times 10^{18} h^{-1} M_{\odot}$ in the cube.

From Figures 3 and 4 it is clear that mass correlation functions and halo correlation functions at different values of $M / M_{*}$ obey the scaling rule quite well. There are some noticeable departures from self-similar scaling in the $M_{*} / 2$ panel at the two earliest output times (especially the earliest), when $M_{*} / 2$ corresponds to 25 and 50 particles, respectively.

The clustering of halos is biased relative to that of the matter. The halo bias factor $b_{h}$ can be defined via $\xi_{h}(r)=b_{h}^{2} \xi_{m}(r)$, where $\xi_{h}$ and $\xi_{m}$ are the two-point correlation functions of halos and matter, respectively. The relation between the clustering of halos and that of the matter has been extensively studied based on analytical models and numerical simulations (e.g., Cole \& Kaiser 1989; Kashlinsky 1991; Mo \& White 1996; Jing 1998, 1999; Porciani, Catelan, \& Lacey 1999; Sheth \& Lemson 1999; Sheth \& Tormen 1999; Sheth, Mo, \& Tormen 2001, and references therein). Mo \& White (1996) give an analytic formula for the bias factor of halos of a given mass based on an extended PressSchechter analysis (Press \& Schechter 1974). This formula is quite accurate for halos with $M>M_{*}$. Jing (1998) empirically modifies the formula to also fit the $N$-body simulation results for halos of lower mass,

$$
\begin{gathered}
b_{h}(M, z)=\left(\frac{1}{2 \nu^{4}}+1\right)^{\left(0.06-0.02 n_{s}\right)}\left(1+\frac{\nu^{2}-1}{\delta_{c, 0}}\right), \\
\nu=\delta_{c}(z) / \sigma(M),
\end{gathered}
$$

where $\sigma(M)$ is the rms fluctuation of the mass density at a mass scale $M, \delta_{c}(z)$ is the threshold density contrast for collapse of a homogeneous spherical overdense region at redshift $z$, and $\delta_{c, 0} \approx 1.69$ for an Einstein-de Sitter universe. For $n_{s}=-1$ here, $\nu=\left(M / M_{*}\right)^{2 / 3}$. We derive the square of the bias factor numerically as a function of $M / M_{*}$ at different redshifts by averaging $\xi_{h}(r) / \xi_{m}(r)$ over comoving separations between 5 and $30 h^{-1} \mathrm{Mpc}$. In this range, the ratio is almost constant. We compare the computed bias factor with that given by Jing's fitting formula in Figure 5. Two conclu- sions can be drawn immediately from the comparison. First, since bias factors at different redshifts overlap with each other when plotted as a function of $M / M_{*}$, the simulation displays the correct scaling behavior. Second, the bias factors agree well with Jing's fitting formula. At higher mass, the computed bias is somewhat lower than that given by Jing's formula. In fact, the same trend can be found in Jing's plot (see his Fig. 2). We attribute this slight deviation in part to a sample volume effect and in part to systematic errors in the approximate analytic formula given by Mo \& White (1996; see Sheth et al. 2001).

Both the GADGET simulation test and the scale-free model test assure that the numerical artifacts in the PM simulations have almost negligible effect on the statistics we care about in this paper. For example, the mass scales that we are concerned with are generally higher than the level where the PM and GADGET halo mass functions begin to deviate slightly. Moreover, the correlation functions are measured at scales $\gtrsim 0.5$ mesh cells, where the PM and GADGET results are consistent. The PM simulations reproduce the expected scaling behaviors in the scale-free model, which also demonstrates that we can measure masses of halos and their spatial distribution correctly based on these simulations. The scaling tests and code comparison suggest that there are some inaccuracies when the halo mass is lower than 50 particles or more than $0.1 \%$ of the total mass in the simulation, but even in these regimes there should be little impact on our conclusions because we compare different cosmologies evolved with the same code and numerical parameters. The PM code is therefore an ideal tool for our purpose in this paper.

\section{COMPARISON OF HALO POPULATIONS}

\subsection{Changing $\Omega_{m}$ with $\sigma_{8}, n_{s}$, and $\Gamma$ Fixed}

We start with a simple case in which we fix the power spectrum (as linearly evolved to $z=0$, with $n_{s}=1.0$, 

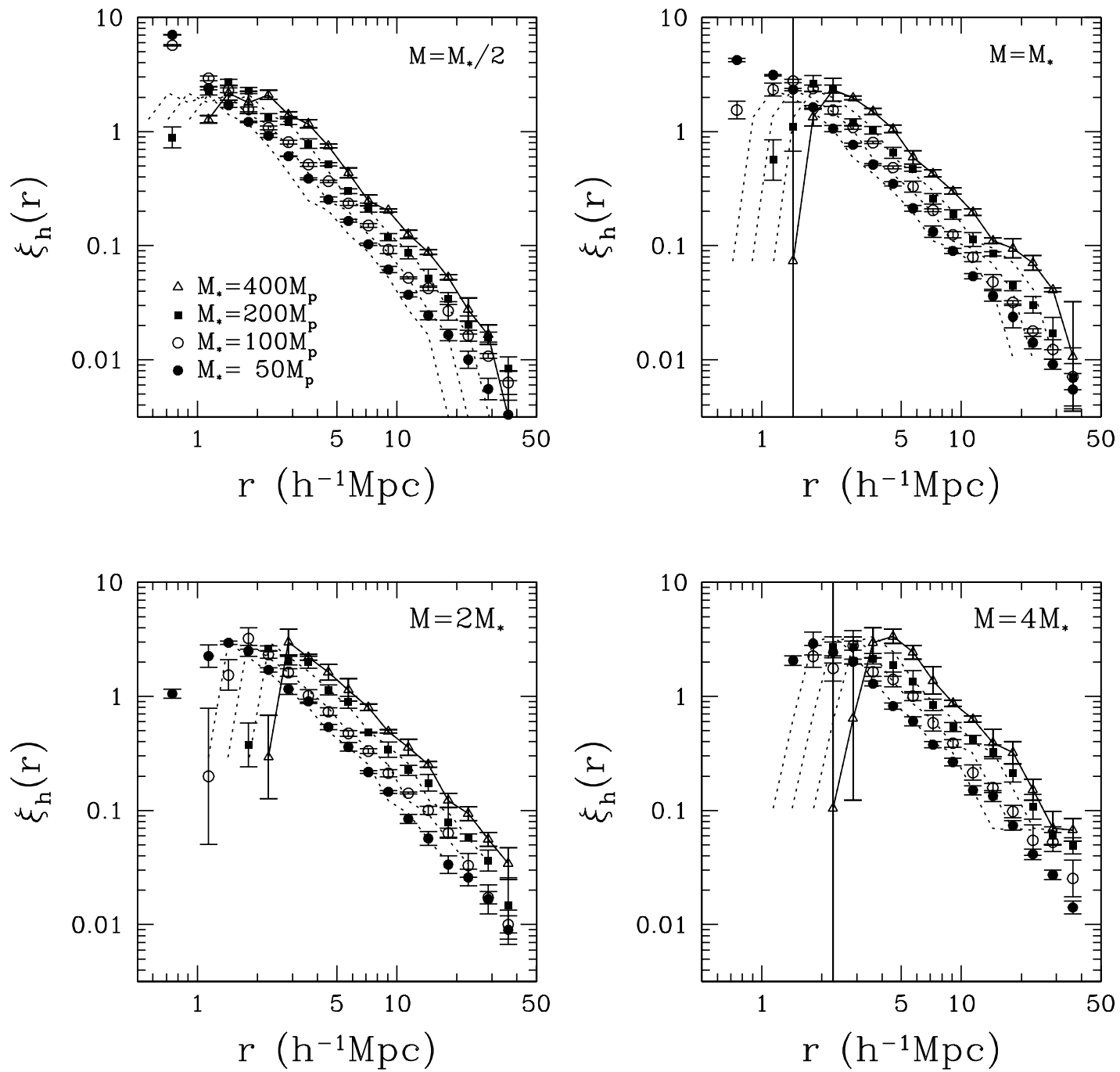

FIG. 4.-Test of self-similar scaling of the halo correlation functions, in mass ranges centered on $M_{*} / 2, M_{*}, 2 M_{*}$, and $4 M_{*}$, as indicated. Points show halo correlation functions at the four evolutionary stages when $M_{*}=50,100,200$, and 400 particles. Dotted lines are obtained by scaling the result of the $M_{*}=400$ output (solid line) to earlier stages, according to eq. (2). Error bars show the dispersion among four independent runs, divided by $\sqrt{3}$.

$\left.\sigma_{8}=0.9, \Gamma=0.20\right)$ and change $\Omega_{m}$. In linear theory, the evolved fluctuations in these models are identical, and this independence of $\Omega_{m}$ holds in the Zeldovich approximation (Zeldovich 1970) as well as its extension, the adhesion approximation, as emphasized by Weinberg \& Gunn (1990). In fact, it holds remarkably well into the fully nonlinear regime, as shown in the top and middle panels of Figure 6, where slices of particle distributions at $z=0$ for models with different $\Omega_{m}$ but identical initial conditions are compared. These slices are taken from GADGET simulations that evolve the particle distribution from $z=19$. Particle distributions of three spatially flat models with different values of $\Omega_{m}$ and one open model with $\Omega_{m}=0.2$ are extremely similar to each other (although particle velocities are higher for higher $\Omega_{m}$ ). Nusser \& Colberg (1998) extend the Zeldovich/adhesion analytic explanation for this similarity of evolved structure by showing that the equations of motion of a collisionless gravitating system of particles in an expanding universe can be put in a form with almost no dependence on $\Omega_{m}$ and $\Omega_{\Lambda}$, if the linear perturbation growth factor is used as the time variable. The evolutionary histories of the four models in Figure 6 are different, but this difference is captured almost entirely by the dependence of the linear growth factor on redshift. The bottom two panels demonstrate this point, showing the particle distributions of the flat $\Omega_{m}=1.0$ and $\Omega_{m}=0.2$ models at the redshifts when the linear growth factor is 0.5 , redshifts $z=1.0$ and $z=1.74$, respectively (we define the growth factor to be unity at $z=0$ ). In most comparisons of $N$-body simulations with different $\Omega_{m}$, the amplitude and/or shape of $P(k)$ is adjusted in concert with $\Omega_{m}$. Such adjustments are well motivated by observational and theoretical considerations, but they mask the fact that the influence of $\Omega_{m}$ at fixed $P(k)$ is extremely simple. Earlier examples of matched comparisons like those in Figure 6 include Figure 1 of Davis et al. (1985) and Figure 5 of Nusser \& Colberg (1998).

Figure 6 suggests that halo mass functions for different models in this case should be nearly the same when measured as a function of particle number, i.e., mass divided by $\Omega_{m}$. This expectation is confirmed in Figure 7, which shows mass functions (from PM simulations) of four spatially flat models. In the left panel, all four (cumulative) mass functions have a similar shape and only shift horizontally relative to each other. When the halo masses are divided by $\Omega_{m}$, 


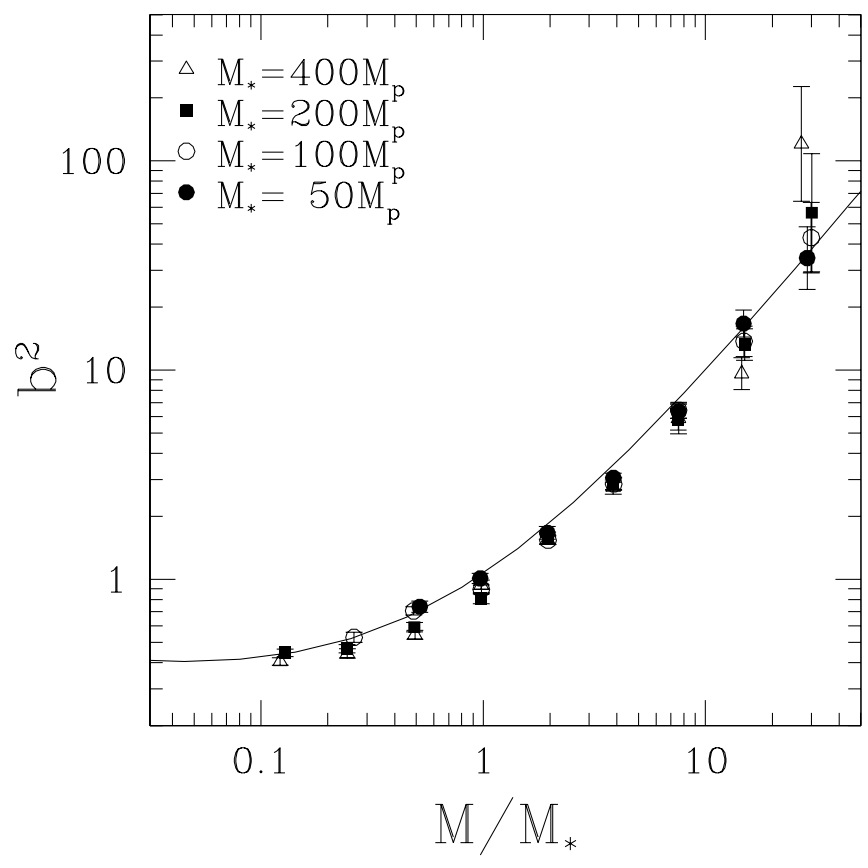

FIG. 5.- Halo bias factor as a function of $M / M_{*}$ in the scale-free simulations. Points show $b^{2}$ at the four evolutionary stages when $M_{*}=50,100$, 200 , and 400 particles. The solid line is calculated using Jing's (1998) formula.

the four curves become nearly identical and overlap with the $\Omega_{m}=1$ curve. To allow a closer inspection, in the right panel we plot the ratio of the scaled mass functions to that calculated using Jenkins et al.'s (2001) fitting formula for the central model. The maximum relative difference between the four scaled mass functions is only about $10 \%$.

The $10 \%$ residual differences reflect slight changes in the characteristic densities and profiles of halos in different cosmologies. For Figure 7, we have identified halos with our standard FOF linking length parameter $b=0.2$, which picks out structures with mean overdensity $\rho / \bar{\rho} \sim 200$ (Davis et al. 1985; Lacey \& Cole 1993), close to the value $\rho / \bar{\rho} \sim 178$ predicted for the post-virialization overdensity in the spherical collapse model for an $\Omega_{m}=1$ universe (Gunn \& Gott 1972; Peacock 1999). If we instead use $b=0.16$, roughly doubling the overdensity threshold $\left(0.2^{3} / 0.16^{3} \approx 1.95\right)$, then halo masses drop by $\sim 20 \%$ as previously linked particles are unlinked, but the level of agreement among different cosmological models stay the same. Another fairly common procedure is to scale $b^{-3}$ in proportion to the virial overdensity predicted by the spherical collapse model, thus using a different $b$ for each cosmological model. If we adopt this approach, taking the virial overdensities from Eke et al. (1996), then the discrepancy of the scaled mass functions reverses sign and rises in amplitude to a maximum $\sim 20 \%$. We conclude that the effect of a pure $\Omega_{m}$ change is described by the simple scaling of halo masses to an accuracy $\sim 10 \%$ for halos defined at fixed overdensity, that the discrepancies reflect the expected cosmology dependence of the characteristic virial overdensity, but that the magnitude of the cosmology correction predicted by the spherical collapse model is too large. Jenkins et al. (2001) also find better agreement among halo mass functions of different cosmological models for halos defined at constant overdensity rather than overdensities scaled according to the spherical collapse model. Henceforth we will use the fixed linking length $b=0.2$ for all models, but our results would not be substantially different if we used $b=0.16$ or scaled $b$ with cosmology.

The mass correlation functions $\xi_{m}(r)$ for these four models are identical at large scales and gradually depart from each other at small scales $\left(r<1 h^{-1} \mathrm{Mpc}\right)$, where the higher overdensities for lower $\Omega_{m}$ boost $\xi_{m}(r)$. We calculate the two-point correlation function for halos of mass $M$ by counting pairs of halos with masses in the range $(M / \sqrt{2}$, $\sqrt{2} M)$. Figure 8 shows halo correlation functions $\xi_{h}(r)$ for four values of $M / M_{*}$. In each case, the four $\Omega_{m}$ models are indistinguishable from each other, as we would expect, since $M_{*}$ scales with $\Omega_{m}$, and comparisons at fixed $M / M_{*}$ therefore remove any dependence on $\Omega_{m}$. The difference in $\xi_{m}(r)$ arises from differences in spatial structure within the halos themselves (which are difficult to discern in Fig. 6). We also calculate the halo bias factor from the mass and halo correlation functions (not shown). When plotted as a function of $M / M_{*}$, the bias factors of the four models are almost the same, as expected, and they agree with Jing's (1998) fitting formula to a similar degree as seen in Figure 5. The halo correlation function plummets at small $r$ because two halo centers cannot be separated by less than the sum of their virial radii. Halos for different $\Omega_{m}$ have the same virial radius at a given $M / M_{*}$ (in simulation terms, the scaling of $M_{*}$ with $\Omega_{m}$ comes from the particle mass, not a change in halo size), so even this exclusion signature is the same in different models.

Although mass functions and spatial clustering of halos are nearly identical as a function of $M / M_{*}$, different values of $\Omega_{m}$ produce different velocity fields. Figure 9 shows the mean pairwise radial (inward) velocity, $v_{12} \equiv$ $-\left(\boldsymbol{v}_{1}-\boldsymbol{v}_{2}\right) \cdot\left(\boldsymbol{r}_{1}-\boldsymbol{r}_{2}\right) /\left|\boldsymbol{r}_{1}-\boldsymbol{r}_{2}\right|$, and the pairwise velocity dispersion, $\sigma_{12} \equiv\left\langle v_{12}^{2}\right\rangle-\left\langle v_{12}\right\rangle^{2}$, at $M=M_{*}$ and $M=8 M_{*}$. Here $\boldsymbol{v}_{i}$ and $\boldsymbol{r}_{i}(i=1,2)$ are the velocities and positions of a halo pair, and the average is over all halo pairs with separations around $r$. Both $v_{12}(r)$ and $\sigma_{12}(r)$ drop as $\Omega_{m}$ drops. The mean pairwise radial velocity $v_{12}(r)$ increases for massive halos and increases much faster for pairs with small separations, a sign of the nonlinear infall velocities induced by high-mass halos. The velocity dispersion $\sigma_{12}(r)$ continues rising out to large separations, and its amplitude at large scales seems to be nearly independent of halo mass. Linear perturbation theory predicts a relation between the peculiar velocity and matter density fields,

$$
\boldsymbol{v}(\boldsymbol{x}) \propto f\left(\Omega_{m}\right) \int \delta(\boldsymbol{x}) \frac{\left(\boldsymbol{x}^{\prime}-\boldsymbol{x}\right)}{\left|\boldsymbol{x}^{\prime}-\boldsymbol{x}\right|^{3}} d^{3} x^{\prime},
$$

where $\delta(\boldsymbol{x})$ is the mass density contrast and $f\left(\Omega_{m}\right) \approx \Omega_{m}^{0.6}$ (see, e.g., Peebles 1993). In the four cosmological models considered here, the mass density contrasts $\delta(\boldsymbol{x})$ should be the same, so linear velocity fields simply scale with $\Omega_{m}^{0.6}$. The dotted curves in Figure 9 show the effect of dividing $v_{12}(r)$ and $\sigma_{12}(r)$ by $\Omega_{m}^{0.6}$; the agreement of these curves with each other and with the $\Omega_{m}=1$ curve demonstrates that the impact of a pure $\Omega_{m}$ change on halo peculiar velocities is well described by the linear theory scaling even on nonlinear scales.

Figure 6 suggests that the agreement of appropriately scaled halo mass functions, correlation functions, and pairwise velocity statistics should hold at other epochs for which the linear growth factors are equal. Although we do not 

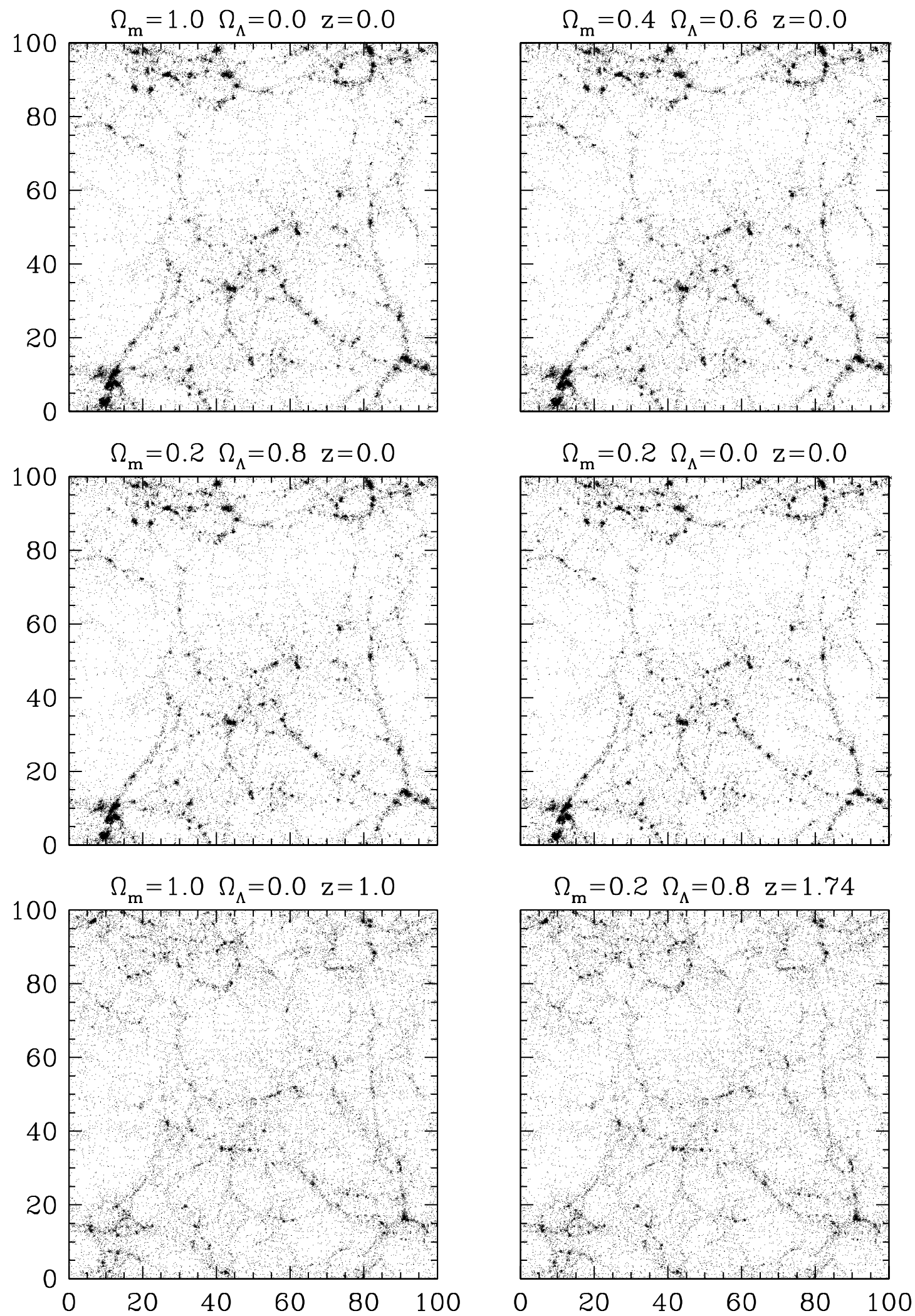

FIG. 6. - Insensitivity of dark matter clustering to the values of $\Omega_{m}$ and $\Omega_{\Lambda}$. The top and middle panels show slices of particle distributions at $z=0$ from simulations that have identical linear theory $P(k)$ and Fourier phases but different combinations of $\Omega_{m}$ and $\Omega_{\Lambda}$, as indicated. The two bottom panels are slices for models $(1.0,0.0)$ and $(0.2,0.8)$ at redshifts corresponding to a linear growth factor of 0.5 . All slices have the size of $100 \times 100 \times 10 h^{-1} \mathrm{Mpc}^{3}$. All simulations were performed with GADGET using $100^{3}$ particles in a $\left(100 h^{-1} \mathrm{Mpc}\right)^{3}$ volume and a force resolution of $\epsilon=0.3 h^{-1} \mathrm{Mpc}$. 

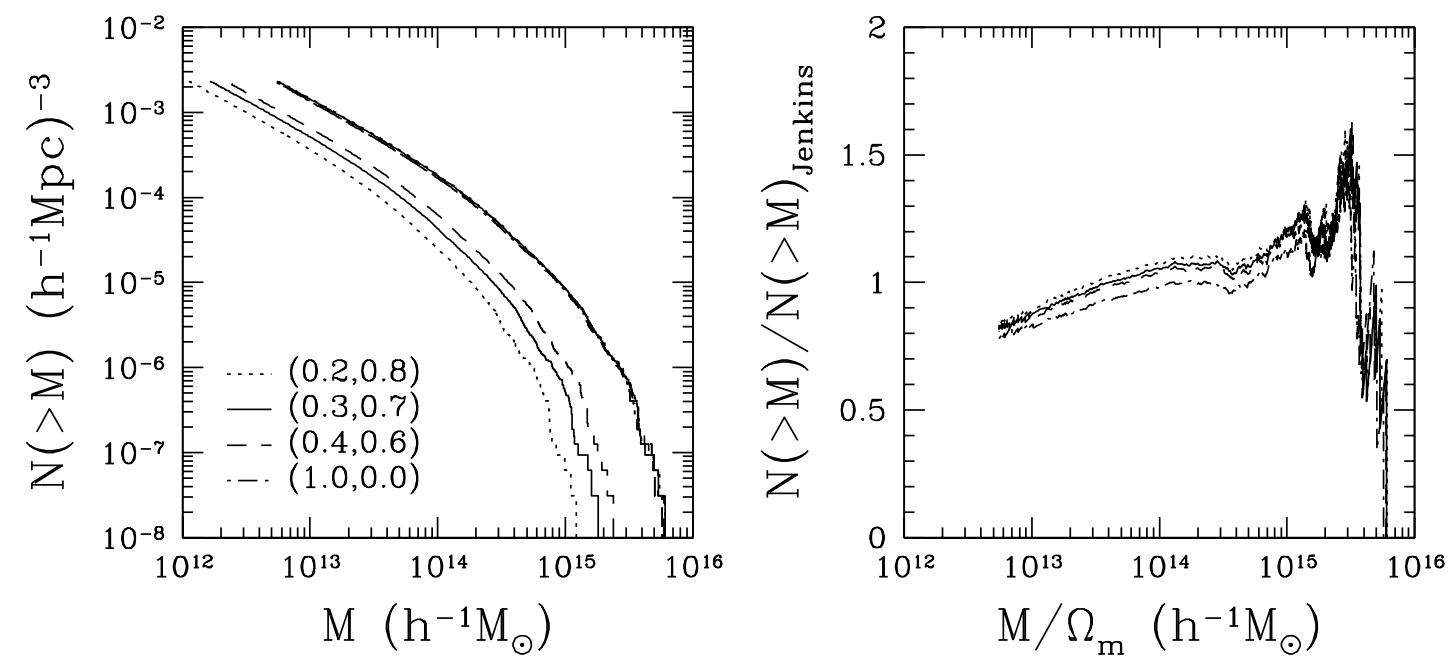

FIG. 7.-Influence of $\Omega_{m}$ on the halo mass function. The left panel shows cumulative halo mass functions from simulations with four different combinations of $\left(\Omega_{m}, \Omega_{\Lambda}\right)$ as indicated. A second curve for each low- $\Omega_{m}$ model shows the result of dividing halo masses by $\Omega_{m}$ before computing $N(>M)$. These scaled curves are almost perfectly superposed on the solid curve representing the $\Omega_{m}=1.0$ model, demonstrating that a change to $\Omega_{m}$ alone simply shifts the mass scale of the halo mass function. The right panel allows closer inspection of this result, suppressing dynamic range by dividing each mass function by the analytic fitting formula of Jenkins et al. (2001) computed for the parameters of the central model $\left(\Omega_{m}=0.3, \Omega_{\Lambda}=0.7\right)$.
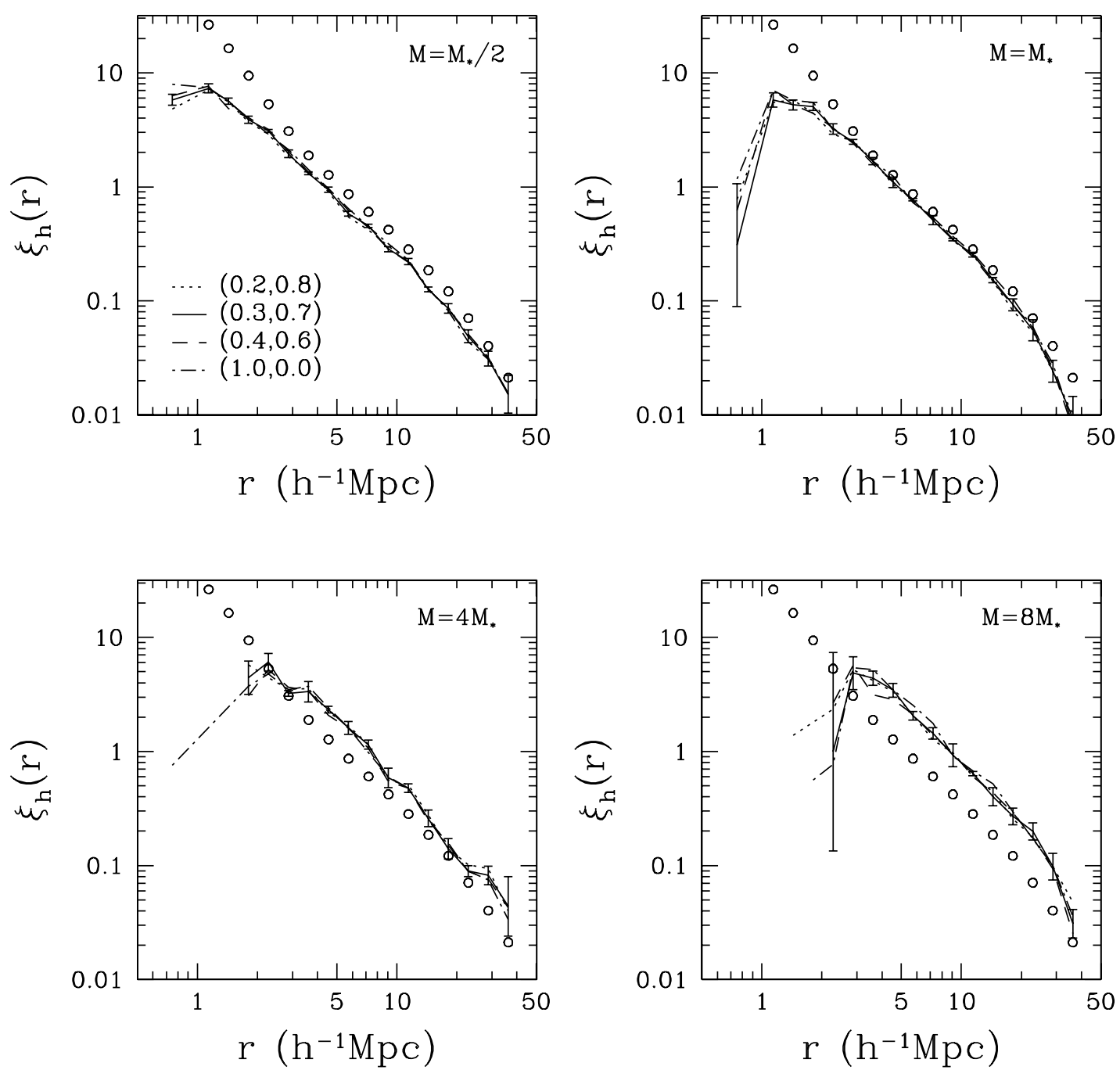

Fig. 8.- Influence of $\Omega_{m}$ on the halo correlation function, for halos in mass ranges centered on $M_{*} / 2, M_{*}, 4 M_{*}$, and $8 M_{*}$ as indicated. Curves in each panel, nearly superposed, show $\xi_{h}(r)$ for the four indicated combinations of $\left(\Omega_{m}, \Omega_{\Lambda}\right)$. To preserve visual clarity, we plot error bars only on the central $(0.3$, 0.7) model, but error bars for other models have similar magnitude. In each panel, open circles show the mass correlation function of the central model. 

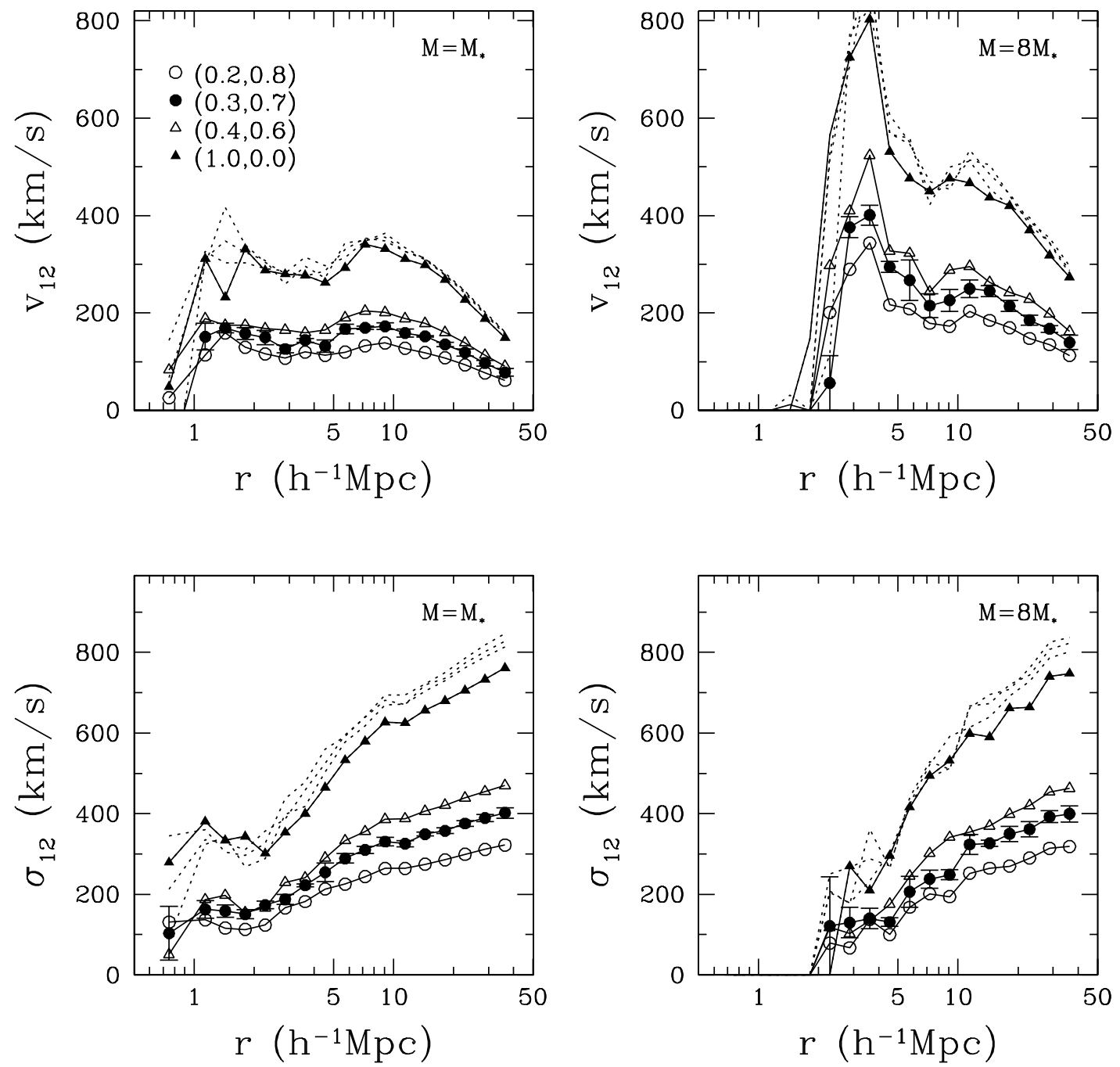

Fig. 9.- Influence of $\Omega_{m}$ on the mean pairwise velocity (top) and the pairwise velocity dispersion (bottom) of halos with mass $M \sim M_{*}$ (left) and $M \sim 8 M_{*}$ (right). Error bars are plotted only for the central model; they increase with $\Omega_{m}$ and are about twice as large for $\Omega_{m}=1$ as for $\Omega_{m}=0.2$. Dotted curves are obtained by dividing the velocity by $\Omega_{m}^{0.6}$; their agreement with the $\Omega_{m}=1$ curve demonstrates that the $\Omega_{m}$ influence is captured almost entirely by the linear theory velocity scaling.

show the results here, we have verified explicitly that the matching of scaled statistical measures in Figures 7, 8, and 9 holds equally well at the redshifts when the four models have growth factors of $0.5(z=1.0,1.36,1.50$, and 1.74 for $\Omega_{m}=1.0,0.4,0.3$, and 0.2 ). Thus, for models with the same $P(k)$ shape and the same $\sigma_{8}$ at $z=0$, the influence of $\Omega_{m}$ and $\Omega_{\Lambda}$ on the evolutionary history of the halo population is entirely encoded in the linear growth factor.

\subsection{Cluster-normalized Models with Fixed P(k) Shape}

Changing $\Omega_{m}$ on its own preserves the shape of the halo mass function but shifts the mass scale in proportion to $\Omega_{m}$. This scaling implies that the threshold volume for collapse is independent of $\Omega_{m}$, given the same power spectrum. If we require that the four cosmological models that differ in $\Omega_{m}$ have halo mass functions that match in amplitude at some physical mass scale (rather than $M / M_{*}$ ), we need to adjust the power spectrum in a way that builds more massive halos in low- $\Omega_{m}$ models and fewer massive halos in high- $\Omega_{m}$ models. In other words, we need a larger threshold volume for collapse in a low- $\Omega_{m}$ model so that its nonlinear mass $M_{*}$ increases. Therefore, if the shape of the power spectrum is kept unchanged, a higher $\sigma_{8}$ is necessary in a lower $\Omega_{m}$ model in order to compensate for the lower mean mass density.

Putting this idea in quantitative form, White, Efstathiou, \& Frenk (1993) argued that the observed abundances of massive clusters of galaxies impose the constraint $\sigma_{8} \approx 0.57 \Omega_{m}^{-0.56}$, with little dependence on the assumed shape of $P(k)$. Many authors have revisited, refined, and recalibrated this "cluster normalization" constraint (see Pierpaoli, Scott, \& White 2001 and references therein). One widely used formulation is that of Eke et al. (1996), who found $\sigma_{8}=(0.52 \pm 0.04) \Omega_{m}^{-0.52+0.13 \Omega_{m}}$ for a spatially flat universe. Inspired by these results, we now consider a sequence of cluster normalized models in which we keep the shape of $P(k)$ fixed but scale the amplitude as $\sigma_{8}=0.9\left(\Omega_{m} / 0.3\right)^{-0.5}$.

Figure 10 shows cumulative halo mass functions for these cluster normalized models. Mass functions of models with $\Omega_{m}=0.2,0.3$, and 0.4 match in amplitude at a cluster scale 

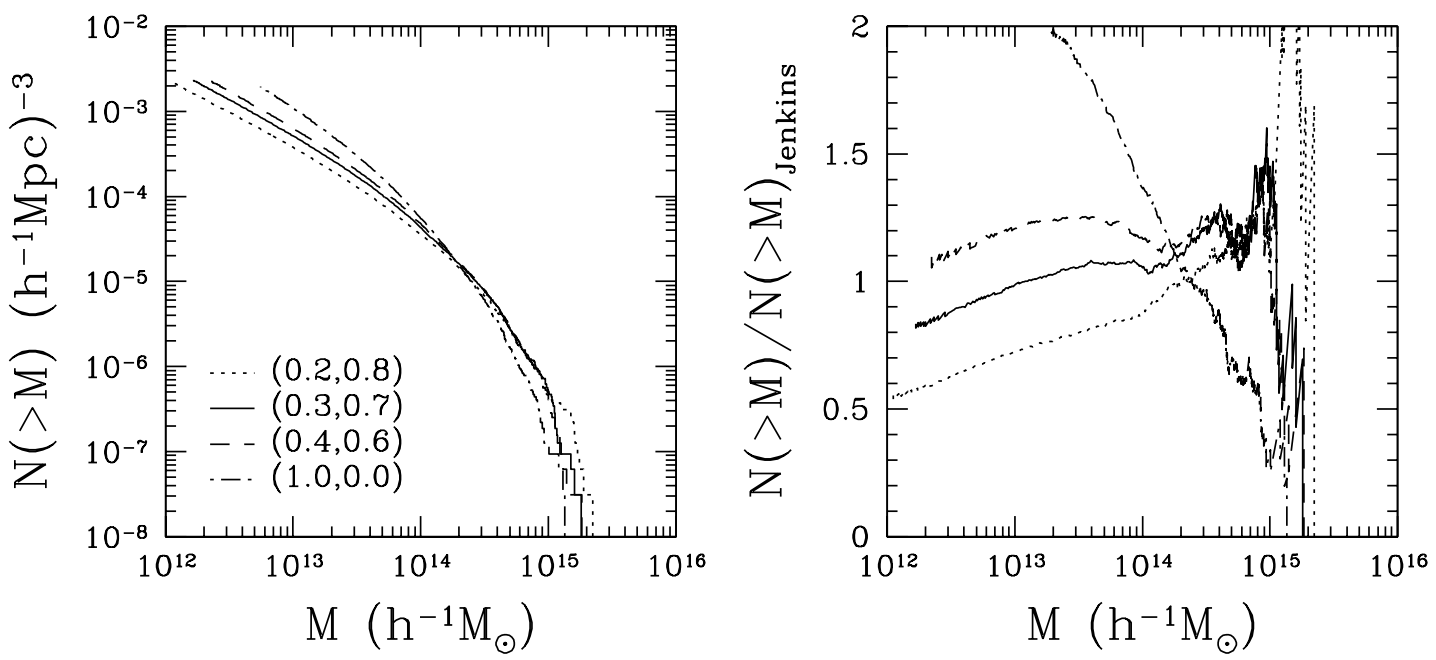

Fig. 10. - Halo mass functions for cluster normalized models, in which the shape of $P(k)$ is fixed but the amplitude is scaled by $\sigma_{8} \propto \Omega_{m}^{-0.5}$. The left panel shows halo mass functions from simulations with four different $\left(\Omega_{m}, \Omega_{\Lambda}\right)$, as indicated. In the right panel, these mass functions are divided by the Jenkins et al. (2001) fitting formula for the central $(0.3,0.7)$ model.

$\left(M \sim 5 \times 10^{14} h^{-1} M_{\odot}\right)$. The mass function of the $\Omega_{m}=1$ model crosses the others at a somewhat lower mass scale, $M \sim 2 \times 10^{14} h^{-1} M_{\odot}$. The discrepancy in matched mass scale just shows that the $\sigma_{8} \propto \Omega_{m}^{-0.5}$ scaling does not hold all the way to $\Omega_{m}=1$, and the Eke et al. (1996) formula indeed implies that we should adopt a somewhat higher $\sigma_{8}$ for $\Omega_{m}=1$. However, the crucial result for our purposes is that the mass functions of these cluster normalized models have systematically different shapes, and there is no value of $\sigma_{8}$ we could choose that would make the $\Omega_{m}=1$ model match the others at more than a single mass scale. Over the smaller range $\Omega_{m}=0.2-0.4$, the cluster normalization condition keeps the halo mass functions reasonably well aligned over about a decade in mass, but by $M \sim 10^{13} h^{-1} M_{\odot}$ the halo space densities of the $\Omega_{m}=0.2$ and 0.4 models differ from those of the central model by $\sim 30 \%$, and from each other by nearly a factor of 2 .

The reason that cluster normalization does not preserve the shape of the halo mass function is that raising $\sigma_{8}$ increases $M_{*}$ but simultaneously drives down the space density of $M_{*}$ clusters by increasing the scale of nonlinearity. For models with the same $P(k)$ shape and the same value of $M_{*}$, the space density of halos at fixed $M / M_{*}$ is proportional to $\Omega_{m}$ (see eq. [7] below). The mass functions of cluster normalized models with different $\Omega_{m}$ must therefore cross at different values of $M / M_{*}$, and because the shapes remain the same as a function of $M / M_{*}$, they cannot stay aligned as a function of $M$.

In these cluster normalized models, the amplitude of $\xi_{m}(r)$ increases as $\Omega_{m}$ decreases, as shown in the upper left panel of Figure 11. At large scales, where the mass density field is still linear, $\xi_{m}(r)$ is just proportional to the amplitude of its Fourier transform, the linear power spectrum, and hence to $\sigma_{8}^{2}$. The roughly constant logarithmic offset in Figure 11 shows that this multiplicative scaling holds approximately even into the strongly nonlinear regime. However, the remaining panels of Figure 11 show that the halo correlation functions are very similar at a fixed physical mass. Qualitatively, this similarity makes sense, since a given mass scale corresponds to a higher value of $M / M_{*}$ when $\Omega_{m}$ is higher, and the correspondingly higher halo bias tends to compensate for the lower mass clustering amplitude. However, it is impressive just how well this cancellation between mass clustering and halo bias works quantitatively. The halo exclusion signature does shift to a larger scale for lower $\Omega_{m}$ because of the larger virial radii needed to compensate for the lower mass density.

Figure 12 shows that the mean pairwise velocity and pairwise dispersion increase with $\Omega_{m}$ in this cluster normalized sequence, but the dependence is weak, especially at large separations. As in Figure 9, more massive halos have higher pairwise velocities, especially at small separations. The weak dependence on $\Omega_{m}$ at large scales can be understood with the help of equation (4). In linear theory, velocity is proportional to $f\left(\Omega_{m}\right) \sigma_{8}$, which, when combined with the cluster normalization condition, reduces to approximately $\Omega_{m}^{0.1}$. Nonlinear evolution at small separations amplifies the difference between models, but only slightly.

\subsection{Matching Mass Functions}

By varying the amplitude of the power spectrum with $\Omega_{m}$, we can match the amplitudes of the cumulative halo mass functions at a given mass scale. However, this combination of parameter changes does not guarantee a match between the shapes of the mass functions at that mass scale. In order to match both the amplitude and the shape of the halo mass functions, we must adjust the relative amplitudes of fluctuations at different scales, or, in other words, the shape of $P(k)$. We therefore need to determine the appropriate combinations of parameters $\left(\sigma_{8}\right.$ and parameters related to the shape of the power spectrum) for each $\Omega_{m}$ model so that all models produce halo mass functions that agree in both amplitude and shape.

\subsubsection{Analytic Solution to Parameter Combinations}

Instead of searching the parameter space to obtain the right power spectrum combinations, we make use of the analytic form of the halo mass function. There are three commonly used analytic formulae for halo mass functions. Press \& Schechter (1974) developed the first theoretical framework based on the spherical collapse model (also see 

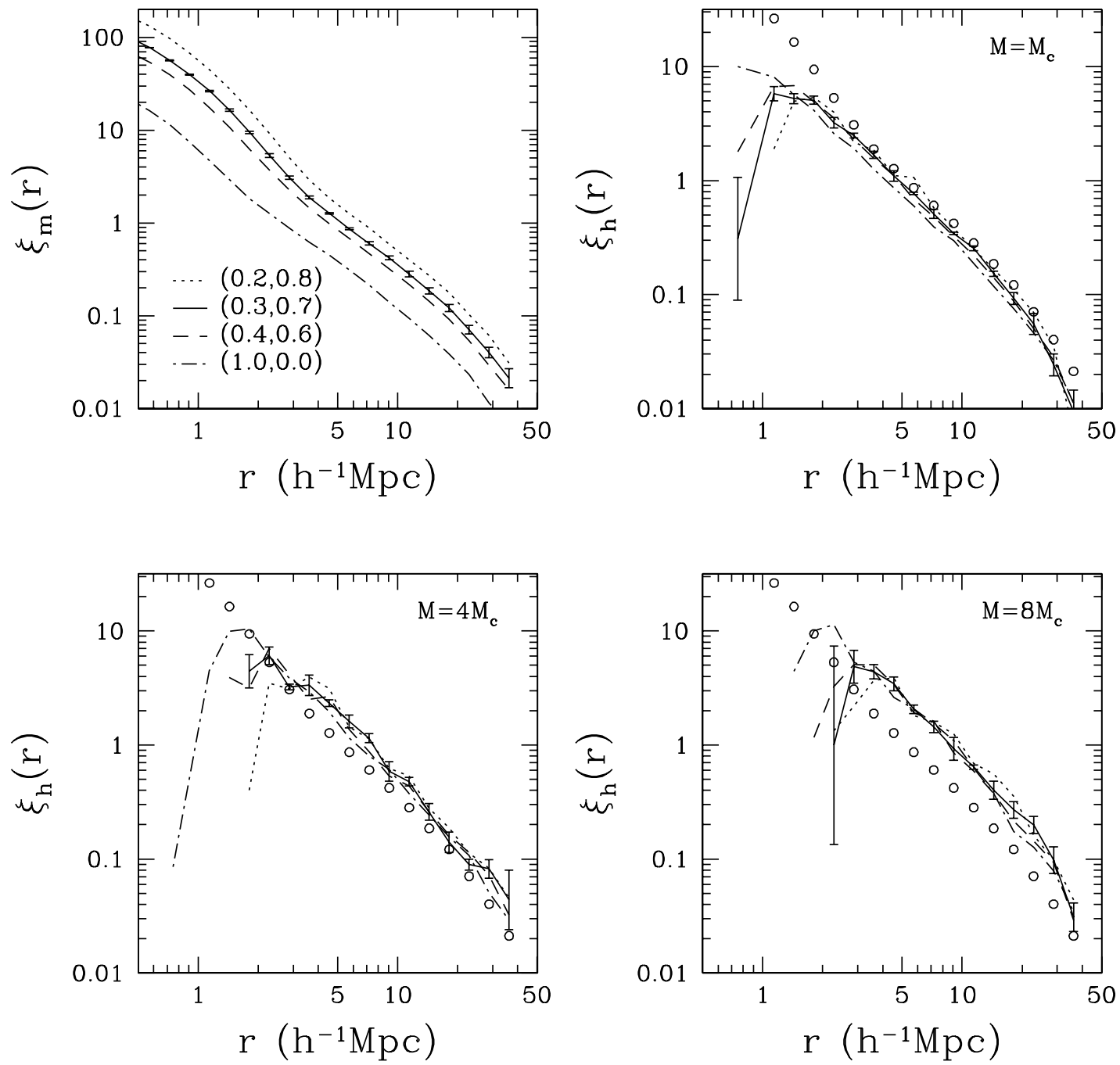

FIG. 11.-Mass and halo correlation functions for cluster normalized models. The upper left panel shows the mass correlation function. The remaining three panels show correlation functions of halos in mass ranges centered on $M_{c}, 4 M_{c}$, and $8 M_{c}$, where $M_{c}=1.03 \times 10^{13} h^{-1} M_{\odot}$ corresponds to $M_{*}$ of the central $(0.3,0.7)$ model. Open circles show the mass correlation function for the central model. Error bars are plotted for the central model and have similar magnitude for other models.

Bond et al. 1991) and provided the first theoretical formula (PS formula) for the halo mass function, which is still widely used. The PS formula is known to underestimate the halo abundance in the high-mass tail (see Jenkins et al. 2001 and references therein). Sheth \& Tormen (1999) give an analytic formula (ST formula) obtained by fitting to the results of $\mathrm{N}$ body simulations, which turns out to be consistent with the ellipsoidal collapse model (Sheth et al. 2001). On the basis of more simulation results, Jenkins et al. (2001) provide the most accurate fitting formula for halo mass functions. However, this formula includes an absolute value of a function, which makes it difficult to use for our present purpose: obtaining an analytic solution of parameter combinations that lead to a good match in halo mass functions. We therefore use the slightly less accurate ST formula.

In the notation of Jenkins et al. (2001), the mass function is defined in terms of the dimensionless function

$$
f(\nu) \equiv \frac{M}{\rho_{0}} \frac{d n(M)}{d \ln \sigma^{-1}},
$$

and the ST formula for the halo mass function can be expressed as (Sheth \& Tormen 1999)

$f(\nu)=A \sqrt{\frac{2 a}{\pi}}\left[1+\left(a \nu^{2}\right)^{-p}\right] \exp \left(\frac{-a \nu^{2}}{2}\right), \nu=\frac{\delta_{c}}{\sigma(M)}$,

where $n(M)$ is the abundance of halos with mass less than $M, \delta_{c} \approx 1.69$ is the threshold density contrast for collapse, $\sigma(M)$ is the rms fluctuation of the mass density at a mass scale $M, \rho_{0}$ is the mean density of the current universe, $A=0.3222, a=0.707$, and $p=0.3$. The mass function can be written as

$$
\frac{d n}{d \ln M}=\frac{d n}{d \ln \sigma^{-1}} \frac{d \ln \sigma^{-1}}{d \ln M}=\alpha \frac{\rho_{0}}{M} f(\nu),
$$

where $\alpha=d \ln \sigma^{-1} / d \ln M=\left(3+n_{\text {eff }}\right) / 6$ characterizes the local index $n_{\text {eff }}$ of the power spectrum and is determined by the shape of the power spectrum. The slope of the mass function is

$\frac{d}{d \ln M}\left(\frac{d n}{d \ln M}\right)=\frac{d n}{d \ln M}\left(\alpha \frac{d \ln f}{d \ln \nu}-1+\frac{d \ln \alpha}{d \ln M}\right)$. 

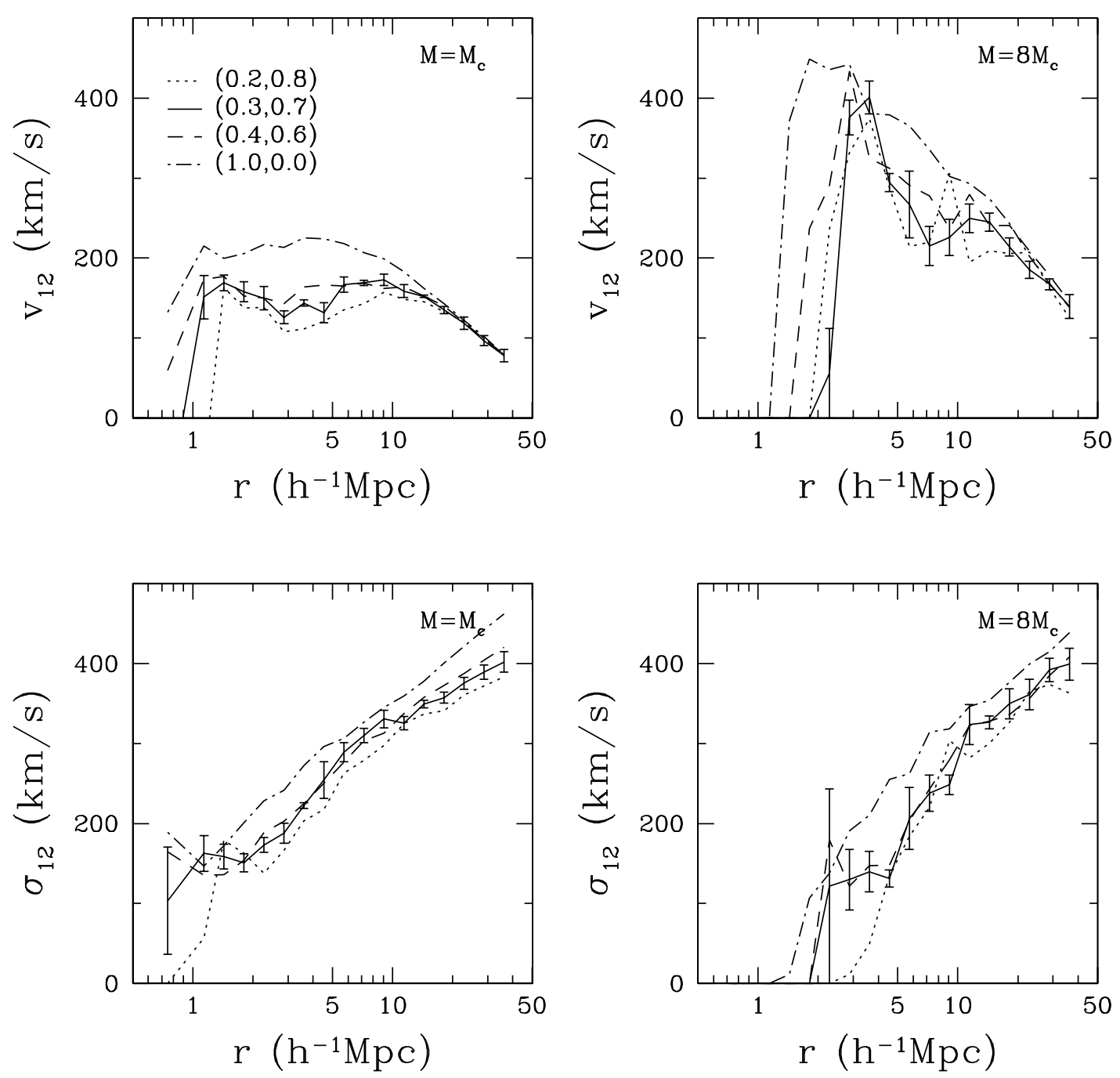

FIG. 12. - Halo velocity statistics for cluster normalized models. Top panels show the mean pairwise radial velocities for halos with $M \sim M_{c}$ (left) and $M \sim 8 M_{c}$ (right), where $M_{c}=1.03 \times 10^{13} h^{-1} M_{\odot}$ corresponds to $M_{*}$ of the central model. Bottom panels show pairwise radial velocity dispersions. Error bars are plotted for the central model and have similar magnitude for other models.

We use a power law to locally approximate the power spectrum (so that the last term on the right-hand side of eq. [8] reduces to zero).

We require that the halo mass function of a given cosmological model match as closely as possible that of the central model at some mass scale. In other words, at this mass scale, both the amplitudes (eq. [7]) and the slopes (eq. [8]) of the two halo mass functions should be equal. We thus solve these two equations for two unknowns $(\alpha, \nu)$ for each cosmological model. All the information contained in the power spectrum is fully embedded in $(\alpha, \nu)$. Therefore, by solving for these two parameters at a given mass scale, we can also determine the normalization $\sigma_{8}$ and shape of the power spectrum.

The power spectrum can be expressed as $P(k) \propto$ $k^{n_{s}} T^{2}(k ; \Gamma)$, where $n_{s}$ is the spectral index of the inflationary power spectrum and $T(k ; \Gamma)$ is the transfer function with shape parameter $\Gamma$. A change in either $n_{s}$ or $\Gamma$ leads to a change in the shape of the power spectrum. We first keep $\Gamma$ fixed and only tilt $P(k)$. Since $\alpha=\left(3+n_{\text {eff }}\right) / 6$ and $n_{\text {eff }}$ is simply the sum of $n_{s}$ and an index given by the transfer function, it is straightforward to obtain the change in $n_{s}$ with respect to that of the central model: $\Delta n_{s}=6 \Delta \alpha$. Once the shape of $P(k)$ is determined, the normalization $\sigma_{8}$ can be obtained from $\nu$. Assuming that mass functions are matched against that of the central model at a cluster mass scale $M=5 \times 10^{14} h^{-1} M_{\odot}$, we calculate $n_{s}$ and $\sigma_{8}$ as a function of $\Omega_{m}$. The left panel of Figure 13 shows that both $n_{s}$ and $\sigma_{8}$ increase sharply toward low $\Omega_{m}$ and drop slowly toward high $\Omega_{m}$. In order to match with the halo mass function of the central model, we need $n_{s}=1.33,0.82,0.42$ and $\sigma_{8}=1.16,0.78,0.55$ for models with $\Omega_{m}=0.2,0.4$, and 1.0 , respectively.

Alternatively, we can keep the spectral index $n_{s}$ fixed $\left(n_{s}=1\right.$ in our calculation) and change the shape parameter $\Gamma$. The right panel of Figure 13 shows $\Gamma$ as a function of $\Omega_{m}$ when halo mass functions are matched at $M=5 \times$ $10^{14} h^{-1} M_{\odot}$. The shape parameter $\Gamma$ also has a sharp increase toward low $\Omega_{m}$ and a slow drop toward high $\Omega_{m}$. For models with $\Omega_{m}=0.2,0.4$, and 1.0 , solutions to $\Gamma$ are $0.35,0.14$, and 0.06 , respectively. In this case, the normalization $\sigma_{8}$ of each model is almost identical to that of the tilted model, which indicates that the two ways of changing the shape of the power spectrum are equivalent at this mass scale. Although pure- $n_{s}$ and pure- $\Gamma$ changes alter the power spectrum in different ways, they produce power spectra (and 

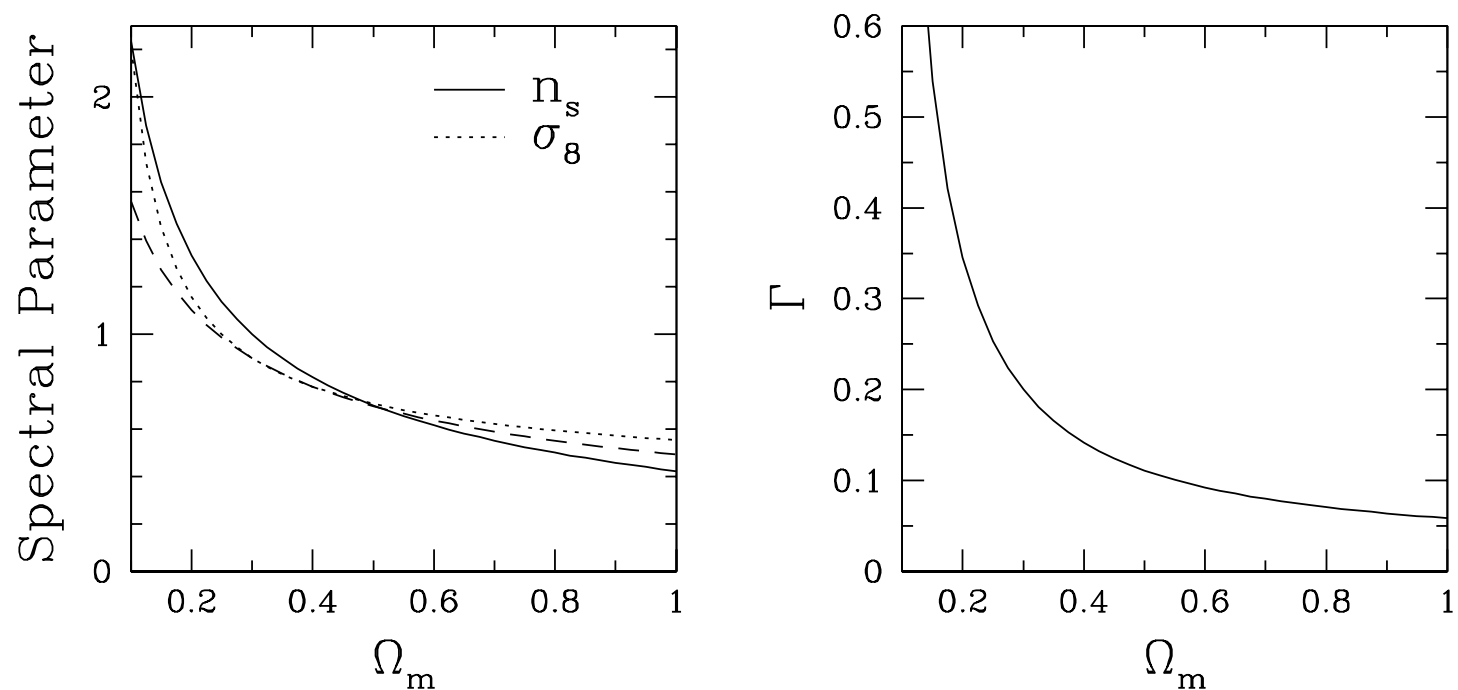

Fig. 13. - Changes in power spectrum parameters required to match the amplitude and slope of the halo mass function at $M=5 \times 10^{14} h^{-1} M_{\odot}$. The left panel shows the values of $\sigma_{8}$ (dotted line) and $n_{s}$ (solid line) required to match the mass function of a model with $\Omega_{m}=0.3, \sigma_{8}=0.9, n_{s}=1.0$, and $\Gamma=0.2$, when $\Gamma$ is fixed at 0.2 . The values of $\sigma_{8}$ we adopt for cluster-normalized models with fixed $P(k)$ shape are plotted as a dashed line, for comparison. The right panel shows the required value of $\Gamma$ if $n_{s}$ is held fixed at 1.0; the corresponding values of $\sigma_{8}$ are nearly identical to those in the left panel. Curves are calculated using the Sheth-Tormen (1999) analytic mass function, as discussed in the text.

thus mass functions) that match closely over a fairly large dynamic range, covering most of the regime in which $\sigma$ is on the order of unity. The near degeneracy can be broken by examining the low-mass end of the halo mass functions. More generally, we could allow both $n_{s}$ and $\Gamma$ to change. In that case, for each value of $\Omega_{m}$, there would be a one-dimensional locus in the $n_{s}$ - $\Gamma$ plane along which models would satisfy the requirement of matching the central model's halo mass function at some mass scale. However, we expect that results from this more general case would be similar to those of the two simple cases.

\subsubsection{Simulation Results}

Using the power spectrum parameters given by the above analytic solutions, we run simulations and make comparisons with the central model. Here we only show results for different tilt models, since models with different values of $\Gamma$ give similar results.

Figure 14 shows cumulative halo mass functions for the four cosmological models. The mass functions agree with each other quite well over a large mass range (from $\sim 10^{13}$ to $\sim 10^{15} h^{-1} M_{\odot}$ ), with only $\sim 10 \%$ relative differences. We do not expect the cumulative mass functions to match exactly, in amplitude and slope at $M=5 \times 10^{14} h^{-1} M_{\odot}$, for three reasons. First, the ST formula is not a perfect fit to the halo mass functions in the simulations. Second, we locally approximate the power spectrum as a power law in order to obtain analytic solutions for the power spectrum parameters. Third, we require a match for differential mass functions instead of cumulative mass functions. Nevertheless, the cumulative mass functions trace each other remarkably well. The nonlinear mass scales are quite different for these four models: $M_{*}=3.10 \times 10^{13}, 1.03 \times 10^{13}, 3.66 \times 10^{12}$,
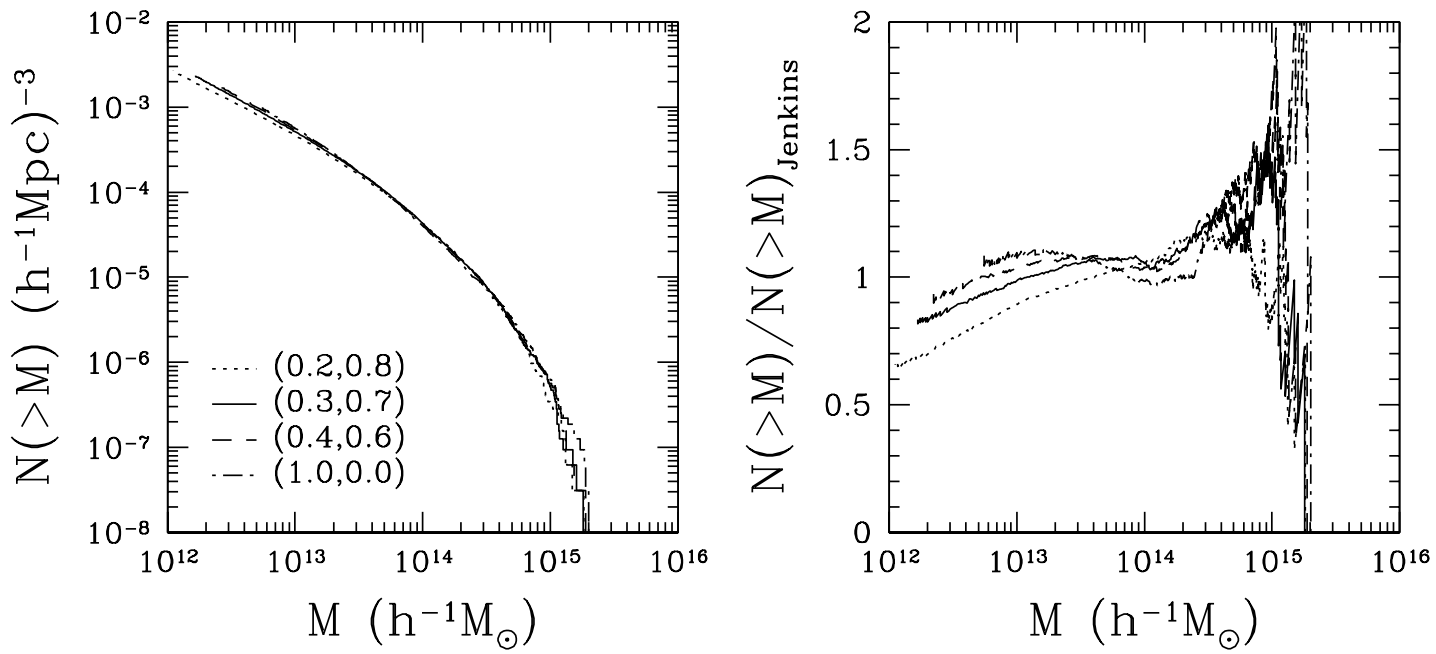

FIG. 14. - Halo mass functions for models that have been matched at $M=5 \times 10^{14} h^{-1} M_{\odot}$ by changing $n_{s}$ and $\sigma_{8}$. As expected, the change in $n_{s}$ yields much better agreement of the mass functions than found for cluster normalized models with no change to the shape of $P(k)$ (see Fig. 10$)$ 
and $7.50 \times 10^{8} h^{-1} M_{\odot}$, for $\Omega_{m}=0.2,0.3,0.4$, and 1.0 , respectively.

Figure 15 shows two-point correlation functions of the mass and halos. Mass is more strongly clustered in low $\Omega_{m}$ models, having a correlation function that is steeper and higher in amplitude than in higher $\Omega_{m}$ models. However, the clustering of halos exhibits the opposite trend. Halos in the $\Omega_{m}=1$ model are highly biased with respect to those of other models, as one might guess from the low nonlinear mass of this model, and this bias more than compensates for the weaker mass clustering. Conversely, at the mass scale $M=1.03 \times 10^{13} h^{-1} M_{\odot}$, shown in the upper right panel of Figure 15, halos in the $\Omega_{m}=0.2$ model are actually antibiased with respect to the mass. Figure 11 shows that for cluster normalized models with fixed $P(k)$ shape, the effects of bias almost exactly cancel the change in $\sigma_{8}$, leaving $\xi_{h}(r)$ nearly independent of $\Omega_{m}$. Since our mass function matching approach imposes a redder $P(k)$ shape (lower $n_{s}$ or $\Gamma$ ) for higher $\Omega_{m}$, and increased large-scale power amplifies the importance of bias, it is not surprising that bias overcompensates mass clustering changes in these models, leaving $\xi_{h}(r)$ steadily dependent on $\Omega_{m}$.
Figure 16 shows the mean pairwise radial velocity and pairwise velocity dispersion for the four cosmological models. As in the case of cluster normalized models, models with higher $\Omega_{m}$ have larger mean pairwise velocities and velocity dispersions than lower $\Omega_{m}$ models. The dependence on $\Omega_{m}$ is stronger and more systematic than that in Figure 12, again a sign of the redder power spectrum of higher $\Omega_{m}$ models.

\section{SUMMARY AND DISCUSSION}

We have examined how changes to the matter density parameter $\Omega_{m}$ and the shape and amplitude of the linear power spectrum $P(k)$ affect the halo mass function, the twopoint halo correlation function, and the first and second moments of the halo pairwise velocity distribution. A change in $\Omega_{m}$, at fixed $P(k)$, simply shifts the halo mass scale. Therefore, if halo masses are scaled in proportion to $\Omega_{m}$, halo populations of different $\Omega_{m}$ models have identical mass functions and clustering properties. However, mean pairwise velocities and pairwise velocity dispersions, which scale as $\Omega_{m}^{0.6}$ on large scales (and fairly far into the nonlinear regime), break this degeneracy. A change of the power spec-
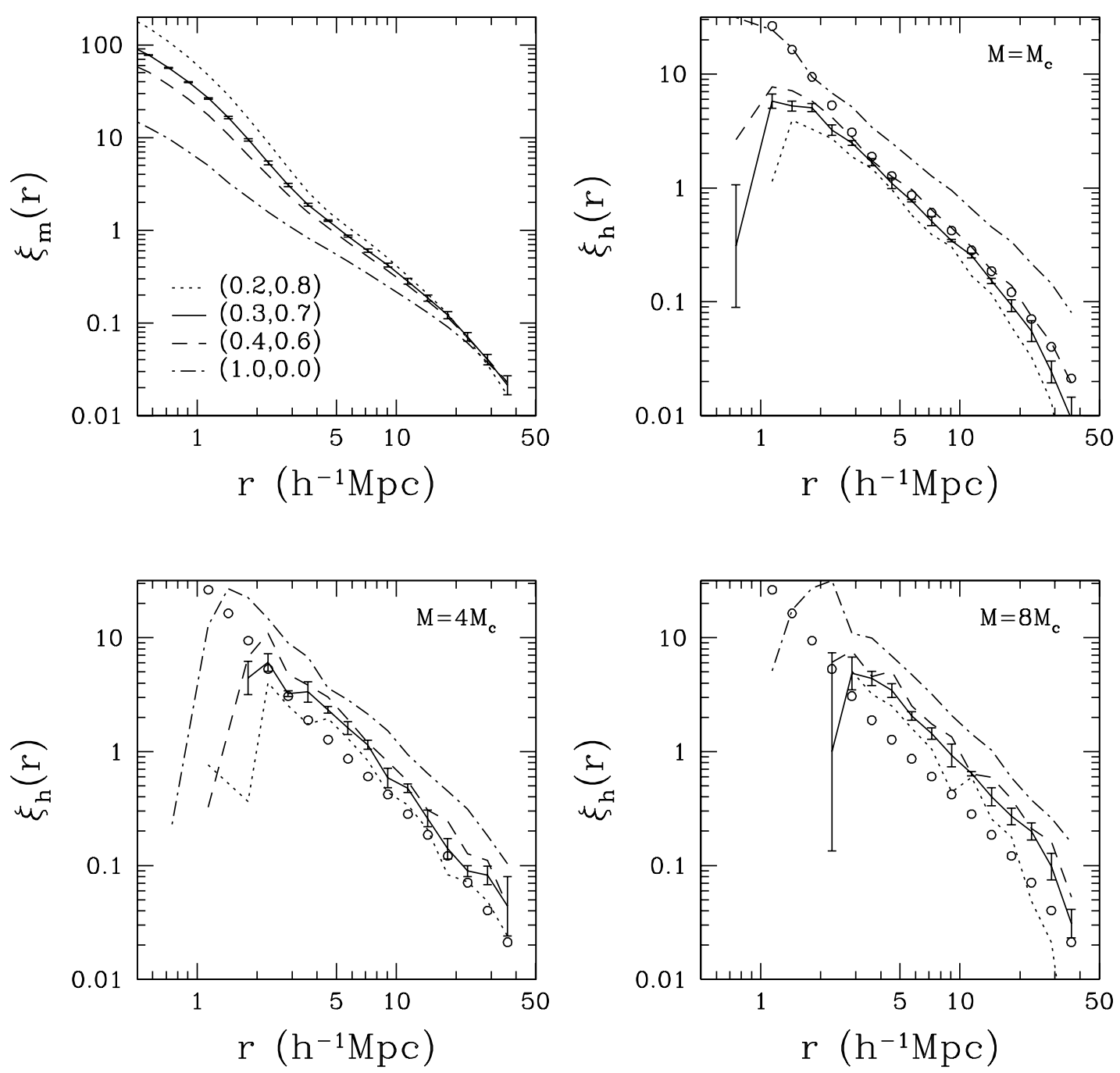

FIG. 15.- Mass and halo correlation functions for the four models of Fig. 14, in the same format as Fig. 11. Halo mass ranges are centered on $M_{c}, 4 M_{c}$, and $8 M_{c}$, where $M_{c}=1.03 \times 10^{13} h^{-1} M_{\odot}$ corresponds to $M_{*}$ of the central model. Open circles in these three panels show the mass correlation function of this model. Error bars are plotted for the central model and have similar magnitude for other models. Changing $n_{s}$ to match the shapes of the halo mass functions leads to substantial differences in halo clustering, in contrast to Fig. 11. 

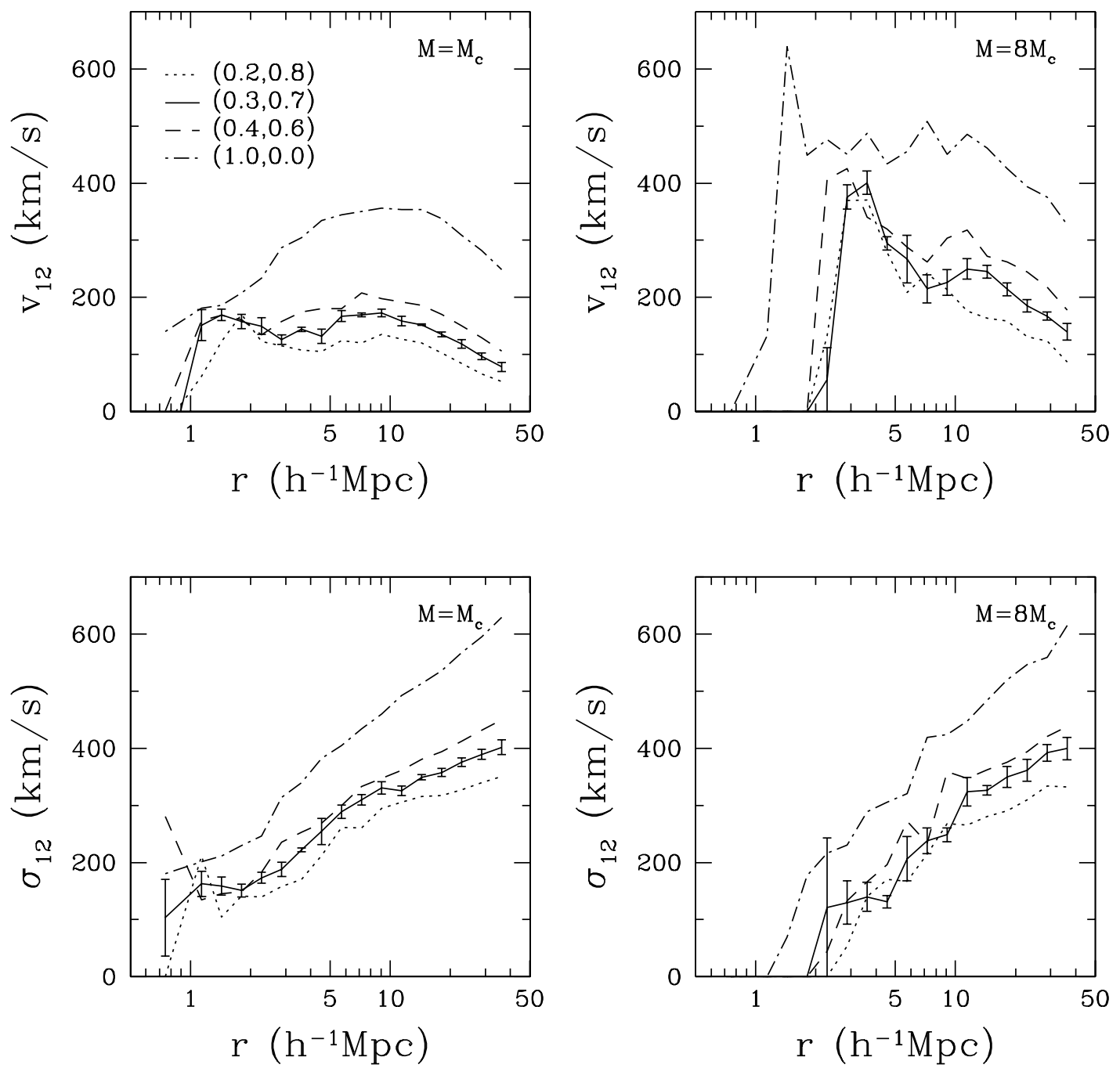

Fig. 16. - Halo velocity statistics for the four models shown in Figs. 14 and 15, in the same format as Fig. 12. Error bars are plotted for the central model; they increase with $\Omega_{m}$ and are about twice as large for $\Omega_{m}=1$ as for $\Omega_{m}=0.2$. Changing $n_{s}$ to match the halo mass function shapes leads to significant systematic differences in the mean pairwise velocities and pairwise velocity dispersions as a function of $\Omega_{m}$, larger than those in Fig. 12.

trum amplitude $\left(\sigma_{8}\right)$ along with $\Omega_{m}$, maintaining a cluster normalization condition $\sigma_{8} \propto \Omega_{m}^{-0.5}$, produces halo populations that have nearly identical spatial clustering at a fixed physical mass scale and systematic but small differences in the mean pairwise velocities and pairwise velocity dispersions. However, while these cluster-normalized models have the same halo space density at $M \sim \operatorname{several} \times 10^{14} M_{\odot}$, the shapes of their mass functions are systematically different. If we further allow the shape of the power spectrum to change (either by tilting it or by changing its shape parameter $\Gamma$ ), we can produce halo populations whose mass functions match very well in both amplitude and shape, over a large mass range. However, the changes to the power spectrum shape cause correlation functions, mean pairwise velocities, and velocity dispersions for halos in the same mass range to differ. We conclude that the halo populations produced by distinct cosmological models are not degenerate. If they are indistinguishable by one statistic, they can be told apart using another statistic.

Our results imply that a perfect knowledge of the halo population and its properties would allow us to pin down the underlying cosmological parameters-specifically, the value of $\Omega_{m}$ and the shape and amplitude of $P(k)$-even before bringing in constraints from other cosmological measurements. However, a galaxy redshift survey detects galaxies rather than halos. Could changes to the halo occupation distribution (HOD) mask the differences in the halo populations of different cosmological models? Models that differ only in $\Omega_{m}$ lead to the same halo mass function and halo clustering in terms of $M / M_{*}$, but with $M_{*} \propto \Omega_{m}$. Their halos could thus be populated the same way as a function of $M / M_{*}$ to produce indistinguishable galaxy spatial clustering. However, any dynamically sensitive statisticsvirial masses of clusters and groups, pairwise velocity dispersions, the parameter combination $\Omega_{m}^{0.6} / b$ inferred from redshift-space distortions, the galaxy-mass correlation function from galaxy-galaxy lensing - would distinguish models with different values of $\Omega_{m}$ immediately. Velocity bias within halos could mask some of these changes, but not all of them (Berlind \& Weinberg 2002). Cluster-normalized models with different $\Omega_{m}$ and the same $P(k)$ shape have different halo mass functions, and they would therefore require a different $P(N \mid M)$ in order to keep the galaxy density fixed. This change would likely cause differences in the galaxy clustering even though the halo clustering is similar, since galaxy clustering is highly sensitive to $P(N \mid M)$ (Ber- 
lind \& Weinberg 2002 and references therein). Changing the shape of $P(k)$ by the amount required to match mass function shapes makes substantial differences to the halo clustering and velocities, which seem unlikely to be masked by a change in the HOD.

These speculations suggest that distinct cosmological models, which produce nondegenerate halo populations, cannot have galaxy populations that are indistinguishable in every spatial and velocity statistic, even if one allows complete freedom in the way that galaxies occupy these halos. We reserve a detailed investigation of this issue for future work. If our optimistic speculation holds true, then high- precision measurements of galaxy clustering and galaxygalaxy lensing should impose strong constraints on cosmological models without reliance on a priori models of galaxy bias.

We thank Volker Springel for advice on GADGET and Changbom Park for the use of his PM code. D. W. thanks the Institute for Advanced Study for its hospitality and the Ambrose Monell Foundation for its financial support during the final stages of this work. This work was supported by NSF grant AST 00-98584.

\section{REFERENCES}

Barnes, J., \& Hut, P. 1986, Nature, 324, 446

Benson, A. J., Cole, S., Frenk, C. S., Baugh, C. M., \& Lacey, C. G. 2000,

Lacey, C., \& Cole, S. 1993, MNRAS, 262, 627

MNRAS, 311, 793

Berlind, A. A., \& Weinberg, D. H. 2002, ApJ, 575, 587

Bond, J. R., Cole, S., Efstathiou, G., \& Kaiser, N. 1991, ApJ, 379, 440

Bullock, J. S., Wechsler, R. H., \& Somerville, R. S. 2002, MNRAS, 329, 246

Colberg, J. M., et al. 2000, MNRAS, 319, 209

Cole, S., \& Kaiser, N. 1989, MNRAS, 237, 1127

Davis, M., Efstathiou, G., Frenk, C. S., \& White, S. D. M. 1985, ApJ, 292, 371

Efstathiou, G., Bond, J. R., \& White, S. D. M. 1992, MNRAS, 258, 1

Efstathiou, G., Frenk, C. S., White, S. D. M., \& Davis, M. 1988, MNRAS, 235,715

Eke, V. R., Cole, S., \& Frenk, C. S. 1996, MNRAS, 282, 263

Freedman, W. L., et al. 2001, ApJ, 553, 47

Governato, F., Babul, A., Quinn, T., Tozzi, P., Baugh, C. M., Katz, N., \& Lake, G. 1999, MNRAS, 307, 949

Gunn, J. E., \& Gott, J. R. 1972, ApJ, 176, 1

Hernquist, L., Bouchet, F. R., \& Suto, Y. 1991, ApJS, 75, 231

Jenkins, A., Frenk, C. S., White, S. D. M., Colberg, J. M., Cole, S., Evrard,

A. E., Couchman, H. M. P., \& Yoshida, N. 2001, MNRAS, 321, 372

Jing, Y. P. 1998, ApJ, 503, L9 .1999, ApJ, 515, L45

Jing, Y. P., Mo, H. J., \& Börner, G. 1998, ApJ, 494, 1

Kashlinsky, A. 1991, ApJ, 376, L5

Kauffmann, G., Colberg, J. M., Diaferio, A., \& White, S. D. M. 1999,

MNRAS, 303, 188

Kauffmann, G., Nusser, A., \& Steinmetz, M. 1997, MNRAS, 286, 795

Ma, C., \& Fry, J. N. 2000, ApJ, 543, 503

Marinoni, C., \& Hudson, M. J. 2002, ApJ, 569, 101

Mo, H. J., \& White, S. D. M. 1996, MNRAS, 282, 347

Netterfield, C. B., et al. 2002, ApJ, 571, 604

Nusser, A., \& Colberg, J. M. 1998, MNRAS, 294, 457

Park, C. 1990, MNRAS, 242, 59P

Peacock, J. A. 1999, Cosmological Physics (Cambridge: Cambridge Univ. Press)

Peacock, J. A., \& Smith, R. E. 2000, MNRAS, 318, 1144

Peebles, P. J. E. 1993, Principles of Physical Cosmology (Princeton: Princeton Univ. Press)

Pierpaoli, E., Scott, D., \& White, M. 2001, MNRAS, 325, 77

Porciani, C., Catelan, P., \& Lacey, C. 1999, ApJ, 513, L99

Press, W. H., \& Schechter, P. 1974, ApJ, 187, 425

Pryke, C., Halverson, N. W., Leitch, E. M., Kovac, J., Carlstrom, J. E., Holzapfel, W. L., \& Dragovan, M. 2002, ApJ, 568, 46

Scoccimarro, R., Sheth, R. K., Hui, L., \& Jain, B. 2001, ApJ, 546, 20

Seljak, U. 2000, MNRAS, 318, 203

Sheth, R. K., \& Lemson, G. 1999, MNRAS, 304, 767

Sheth, R. K., Mo, H. J., \& Tormen, G. 2001, MNRAS, 323, 1

Sheth, R. K., \& Tormen, G. 1999, MNRAS, 308, 119

Springel, V., Yoshida, N., \& White, S. D. M. 2001, NewA, 6, 79

Weinberg, D. H., \& Gunn, J. E. 1990, MNRAS, 247, 260

White, M., Hernquist, L., \& Springel, V. 2001, ApJ, 550, L129

White, S. D. M., Efstathiou, G., \& Frenk, C. S. 1993, MNRAS, 262, 1023

York, D. G., et al. 2000, AJ, 120, 1579

Yoshikawa, K., Taruya, A., Jing, Y. P., \& Suto, Y. 2001, ApJ, 558, 520

Zeldovich, Ya. B. 1970, A\&A, 5, 84 